\title{
Stability, Multiplicity and Global Continuation of Symmetric Periodic Solutions of a Nonlinear Volterra Integral Equation
}

\author{
S.-N. Chow, ${ }^{\dagger, *}$ O. Diekmann ${ }^{\dagger \dagger}$ and J. Mallet-PareT ${ }^{\dagger \dagger}{ }^{\dagger+*}$ \\ ${ }^{\dagger}$ Michigan State University, East Lansing, \\ Michigan 48824, U.S.A. \\ ${ }^{+}$Centre for Mathematics and Computer Science, \\ Amsterdam, The Netherlands \\ ${ }^{\dagger+}$ Division of Applied Mathematics, Brown University, \\ Providence, Rhode Island 02912, U.S.A.
}

Received January 21, 1985

Results on existence, multiplicity, stability, global continuation and limiting behaviour when $\varepsilon \downarrow 0$ of periodic solutions of

$$
x(t)=\frac{1}{2 \varepsilon} \int_{1-\varepsilon}^{1+\varepsilon} f(x(t-\tau)) d \tau
$$

are derived for the case of a nonlinear function $f$ having certain monotonicity and symmetry properties. The proofs are based on the following two observations:

(i) the right-hand side of (E) defines an operator which maps a cone of two-periodic functions with symmetry and positivity properties into itself; and

(ii) slowly oscillating solutions of the linear variational equation correspond to dominant Floquet multipliers.

Key words: singular perturbation, global Hopf bifurcation, Volterra convolution integral equation, invariant cone, periodic solutions, stability, slowly oscillating solutions, Floquet multipliers

\section{Introduction}

In this paper we study the nonlinear scalar Volterra convolution integral equation

$$
x(t)=\frac{1}{2 \varepsilon} \int_{1-\varepsilon}^{1+\varepsilon} f(x(t-\tau)) d \tau, \quad 0<\varepsilon<1,
$$

with a nonlinear function $f: \boldsymbol{R} \rightarrow \boldsymbol{R}$ whose graph has the form shown in Fig. 1 .

More precisely we assume that $f \in C^{2}$ and

$$
\begin{aligned}
& H_{1} \quad f(-x)=-f(x) \quad \text { if } \quad x \in(-\infty, \infty), \\
& H_{2} f(1)=-1,
\end{aligned}
$$

\footnotetext{
*) Partially supported by NSF Grant MCS- 8201768 .
} 


$$
\begin{array}{ll}
H_{3} f^{\prime}(x)<0 & \text { if } x \in(-\infty, \infty), \\
H_{4} \quad f^{\prime \prime}(x)>0 & \text { if } x \in(0, \infty) .
\end{array}
$$

Note that these hypotheses imply $f(x)<-x$ on $(0,1)$, and $f^{\prime}(0)<-1$. Frequently we will use the function $g(x)=-f(x)$. Unless stated otherwise, we assume $0<\varepsilon<1$.

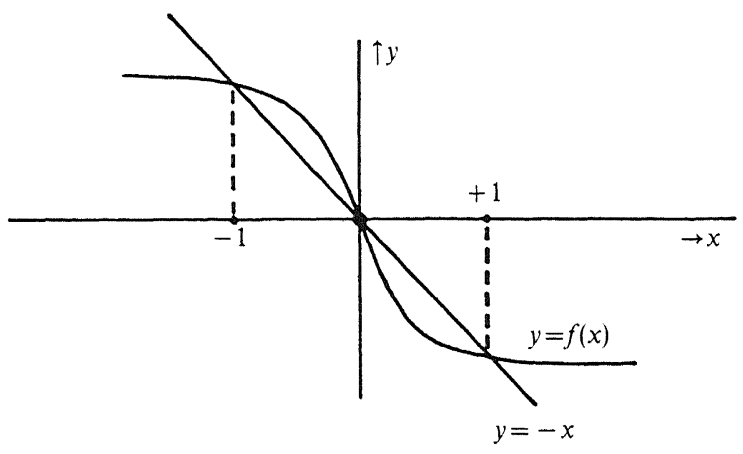

Fig. 1

When $\varepsilon \downarrow 0$ equation (E) formally degenerates into the difference equation

$$
x(t)=f(x(t-1)) .
$$

When $t$ is restricted to the integers $Z$, our assumptions on $f$ imply that (D) has a globally stable periodic solution of period two consisting of the points \pm 1 . Following earlier work by Hoppensteadt [12] on a similar integral equation (also see Cushing [3] and Greenberg [7]), by Hadeler and Tomiuk, Chow, Mallet-Paret and Nussbaum $[2,8,17,19,20]$ on the differential-delay equation $\varepsilon x^{\prime}(t)=-x(t)+f(x(t-1))$, and by Nussbaum and others on related equations (see [25] and the survey article [26]), we address here the problems of existence, stability, continuation and limiting behavior when $\varepsilon \downarrow 0$ of periodic solutions of $(\mathrm{E})$.

Among other things, we will show that $(\mathrm{E})$ admits a family $x_{\varepsilon}(t)$ of periodic solutions of fixed period two. This family arises as a Hopf bifurcation from the zero solution at $\varepsilon=\varepsilon^{*}$ for some $0<\varepsilon^{*}<1$ and exists for $\varepsilon$ in the interval $\left(0, \varepsilon^{*}\right)$. For every $\varepsilon$ in this interval the solution $x_{\varepsilon}$ has the form as shown in Fig. 2. In particular, the symmetry conditions

$$
\begin{aligned}
& x_{\varepsilon}(-t)=-x_{\varepsilon}(t), \\
& x_{\varepsilon}(t+1)=-x_{\varepsilon}(t)
\end{aligned}
$$

both hold, as well as the monotonicity and convexity conditions

$$
\begin{array}{ll}
x_{\varepsilon}^{\prime}(t)>0 & \text { in } \quad\left(-\frac{1}{2}, \frac{1}{2}\right), \\
x_{\varepsilon}^{\prime \prime}(t)<0 \quad \text { in } \quad(0,1) .
\end{array}
$$


The idea to look for periodic solutions in a cone characterized by (1.1), (1.2) and positivity on $(0,1)$ was introduced by Nussbaum [31] in the context of functional differential equations.

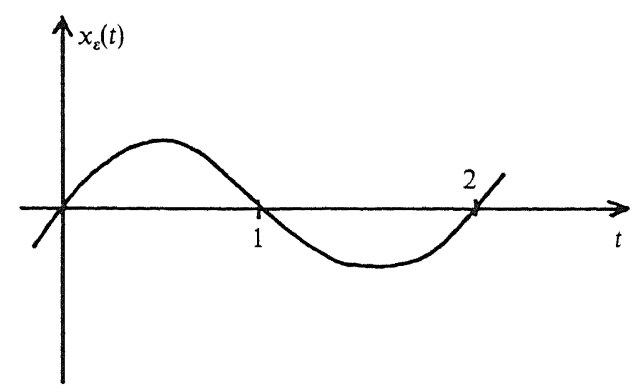

Fig. 2

The solution $x_{\varepsilon}$ is unique among functions satisfying the symmetry conditions (1.1) and (1.2) and which are non-negative on $(0,1)$. Furthermore, the family $x_{\varepsilon}$ depends monotonically on the parameter $\varepsilon$ :

$$
x_{\varepsilon_{1}}(t)<x_{\varepsilon_{2}}(t)<1 \quad \text { if } \quad \varepsilon_{1}>\varepsilon_{2} \text { and } t \in(0,1) .
$$

As $\varepsilon \downarrow 0$ the periodic solution $x_{\varepsilon}$ converges to the "square wave" function

$$
\operatorname{sqw}(t)=\left\{\begin{aligned}
1 & \text { if } t(\bmod 2) \in(0,1) \\
-1 & \text { if } t(\bmod 2) \in(-1,0)
\end{aligned}\right.
$$

uniformly on compact subsets of $(-\infty, \infty)-Z$. Near the discontinuities of $\operatorname{sqw}(t)$ (i.e. near integer values of $t$ ) the solution $x_{\varepsilon}$ possesses a transition layer of width $O(\varepsilon)$. The precise asymptotic form of this layer is described by the monotone antisymmetric solution of a transition layer equation associated with the equation (E).

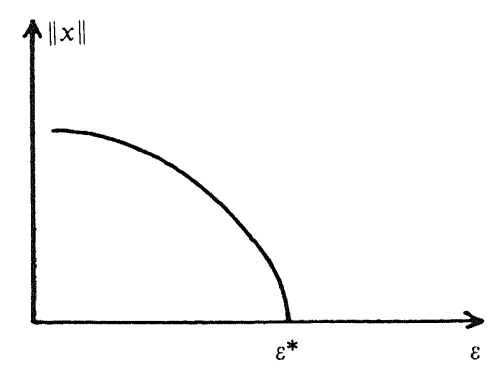

Fig. 3

Figure 3 depicts the global family of solutions $x_{\varepsilon}$ for $0<\varepsilon<\varepsilon^{*}$. Note that we have not yet excluded the possibility of secondary bifurcations of non-symmetric solutions from this family, and such bifurcations, if they exist, are not shown in this Figure. The above results on existence, uniqueness, and asymptotic behavior of $x_{\varepsilon}$, as well as smooth dependence on $\varepsilon$, are proved in Sections 2, 3, 4, 5 and 7 of this paper. 
Our approach is to exploit the symmetry of $f$ in reducing the search for periodic solutions to the search for fixed points of a monotone operator. Subsequently we use well-known techniques due to Krasnosel'skii [16].

The stability of $x_{\varepsilon}$ near the Hopf bifurcation follows from established results based on the direction of bifurcation. These results imply that for $\varepsilon^{*}-\varepsilon$ sufficiently small the solution $x_{\varepsilon}$ is asymptotically stable. We refer to Diekmann and van Gils [4] for a discussion and presentation of a qualitative theory, which includes the Hopf bifurcation problem, for the general class of Volterra convolution integral equations of the form

$$
x(t)=\int_{0}^{\infty} B(\tau) f(x(t-\tau)) d \tau .
$$

Our equation $(E)$ is obtained from (1.3) with the kernel

$$
B(\tau)= \begin{cases}\frac{1}{2 \varepsilon} & \text { if } 1-\varepsilon \leqslant \tau \leqslant 1+\varepsilon, \\ 0 & \text { otherwise } .\end{cases}
$$

Note that the standard Hopf bifurcation results (see Hale [9]) for the differentiated form

$$
x^{\prime}(t)=\frac{1}{2 \varepsilon}[f(x(t-1+\varepsilon))-f(x(t-1-\varepsilon))]
$$

of (E) do not apply, due to the fact that the delays $1 \pm \varepsilon$ vary with the parameter $\varepsilon$ (see, however, the paper of Hale [10]). The fact that the linearization of (1.5) has $\mu=0$ as an additional eigenvalue also poses a problem. We also remark that many of the results we obtain depend on the specific form (1.4) of the kernel $B$, and may not hold for the more general Volterra equation (1.3) with arbitrary kernel $B$.

For values of $\varepsilon$ which are not near the bifurcation point $\varepsilon^{*}$ new global methods must be developed to study the stability of $x_{\varepsilon}$. This is done in Sections 6, 8 and 9. In particular, a theory of "slowly oscillating solutions" is developed in Section 8 and used to prove asymptotic stability of $x_{\varepsilon}$ for all $\varepsilon \in\left(0, \varepsilon^{*}\right) \cap(0,1 / 3]$. (Of course this implies that there are no secondary bifurcations from the branch $x_{\varepsilon}$ in Fig. 3 for this range of $\varepsilon$.) The restriction $\varepsilon \leqslant 1 / 3$ occurs quite naturally, and repeatedly, in our stability proof, in that we require the length $2 \varepsilon$ of the interval of integration in equation (E) not to exceed the "minimum delay" $1-\varepsilon$; that is, $2 \varepsilon \leqslant 1-\varepsilon$. Whether this restriction is essential, or is merely an artifact of the proof, is an open question. We suspect it is the latter, and indeed we conjecture that the solution $x_{\varepsilon}$ is asymptotically stable for all $\varepsilon \in\left(0, \varepsilon^{*}\right)$.

As a historical side remark we mention that the notion of a slowly oscillating solution of a delay equation already appears in the book by Myshkis [24]. Kaplan and Yorke [14,15] and Walther [28] established certain stability properties of slowly oscillating solutions.

Other families of periodic solutions are also shown to exist. Some of these are 
obtained by rescaling the known family $x_{\varepsilon}(t)$ : if $k \geqslant 3$ is an odd integer and $\varepsilon<\varepsilon^{*} / k$, then $y(t)=x_{k \varepsilon}(k t)$ is a solution of $(\mathrm{E})$ of period $2 / k$. This gives countably many families of "rapidly oscillating" periodic solutions, arising as Hopf bifurcations from $x=0$ at the points $\varepsilon^{*} / 3>\varepsilon^{*} / 5>\varepsilon^{*} / 7>\cdots$. As $\varepsilon \downarrow 0$ these solutions tend to scaled versions of the square wave. They are analogous to the rapidly oscillating solutions obtained for some differential-delay equations by Chow, Mallet-Paret and Nussbaum $[2,19,20]$.

If $\left|f^{\prime}(0)\right|$ is large enough, there are additional Hopf bifurcations from the zero solution. However, the bifurcating families are absorbed again by the zero solution through another Hopf bifurcation at a smaller (but positive) value of $\varepsilon$. Thus the zero solution acts as both a source and sink for such a family, in accordance with one of the alternatives in the global Hopf Bifurcation Theorem. (See, for instance, [21] and [22].)

All of the families arising from Hopf bifurcations, with the exception of the stable "primary family" $x_{\varepsilon}$ arising at $\varepsilon^{*}$, are unstable whenever $\varepsilon \leqslant 1 / 3$. Our results do not rule out the possibility that (E) admits other periodic solutions, possibly stable ones, not arising from Hopf bifurcations. Similarly it is unclear whether an arbitrary periodic solution necessarily has period $p$ of the form $p=2 / k$ for some integer $k$.

\section{Hopf Bifurcation}

Linearization of $(\mathrm{E})$ around the constant solution $x \equiv 0$ leads to

$$
z(t)=\frac{f^{\prime}(0)}{2 \varepsilon} \int_{1-\varepsilon}^{1+\varepsilon} z(t-\tau) d \tau .
$$

Upon substitution of $z(t)=e^{\mu t}$ we arrive at the characteristic equation

$$
1=f^{\prime}(0) e^{-\mu} \frac{e^{\varepsilon \mu}-e^{-\varepsilon \mu}}{2 \varepsilon \mu} .
$$

Looking for roots on the imaginary axis we put $\mu=i v$ and find the two real equations

$$
\begin{aligned}
& \frac{2 \varepsilon}{f^{\prime}(0)} v \sin v=0, \\
& \frac{2 \varepsilon}{f^{\prime}(0)} v \cos v=2 \sin \varepsilon v .
\end{aligned}
$$

The first implies $v=k \pi$, where $k \in Z$, which upon substitution into the second leads to

$$
\sin \gamma \pi=(-1)^{k} \frac{\gamma \pi}{f^{\prime}(0)}
$$

where $\gamma=\varepsilon k$. This equation can easily be analysed graphically when we recall that $f^{\prime}(0)<-1$. See Fig. 4. 


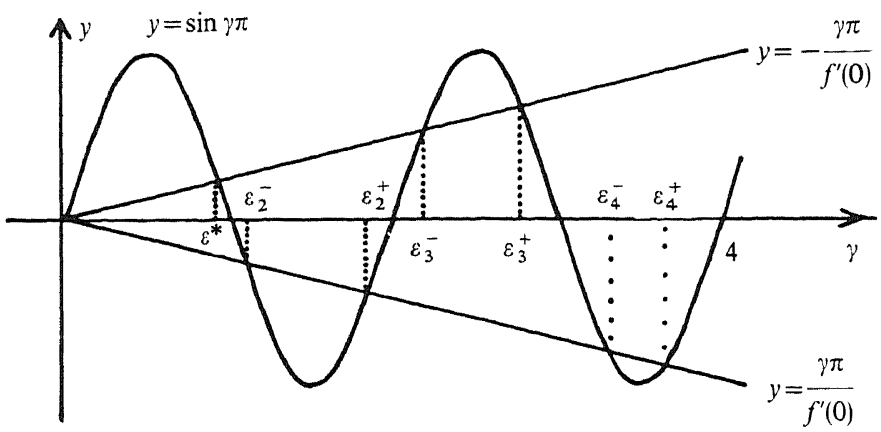

Fig. 4

PROPOSITION 2.1. For odd $k$ equation (2.2) has precisely one root $\gamma=\varepsilon^{*}$ in the interval $(0,1)$, and no root in this interval for even $k$.

In addition there exist universal constants $1<\kappa_{2}<\kappa_{3}<\cdots$ and $\gamma_{n} \in(n-1, n)$ for $n \geqslant 2$ such that when the parities of $k$ and $n$ are the same, each interval $(n-1, n)$ contains

- no roots $\gamma$ if $\left|f^{\prime}(0)\right|<\kappa_{n}$;

- precisely one root $\gamma=\gamma_{n}$ if $\left|f^{\prime}(0)\right|=\kappa_{n}$;

- precisely two roots $\gamma=\varepsilon_{n}^{ \pm}$, with $\varepsilon_{n}^{-}<\gamma_{n}<\varepsilon_{n}^{+}$, if $\left|f^{\prime}(0)\right|>\kappa_{n}$.

The interval $(n-1, n)$ contains no roots $\gamma$ when the parities of $k$ and $n$ are different.

Proof. The proof is straightforward. The constant $\gamma_{n}$ is the unique root of $\tan \gamma \pi=\gamma \pi$ in $(n-1, n)$ while $\kappa_{n}=1 /\left|\cos \gamma_{n} \pi\right|$.

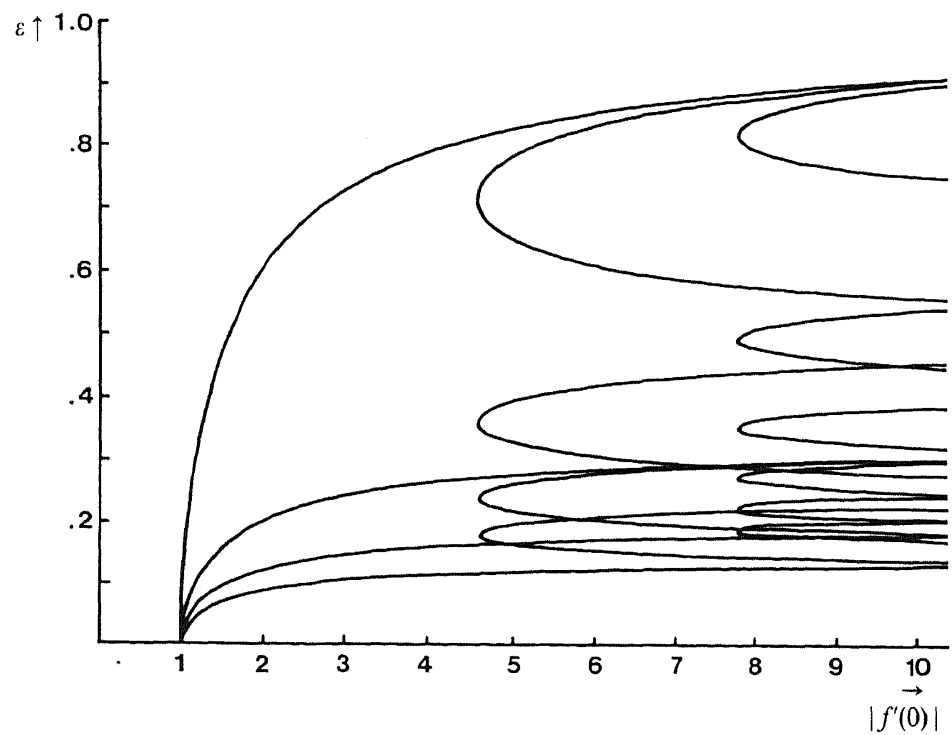

Fig. 5 
Figure 5 depicts the solutions $\varepsilon$ as functions of $f^{\prime}(0)$; that is, the curves $\varepsilon=\varepsilon^{*} / k$ and $\varepsilon=\varepsilon_{n}^{ \pm} / k$ are plotted in the $\left(\left|f^{\prime}(0)\right|, \varepsilon\right)$-plane for various $n$ and $k$. Some simple calculations based on this Figure lead to the following result.

PROPOSITION 2.2. As \& decreases, a simple root crosses the imaginary axis at $\mu=i k \pi$ with non-zero speed

-from left to right as $\varepsilon$ passes through $\varepsilon^{*} / k$, for $k$ odd;

-from left to right as $\varepsilon$ passes through $\varepsilon_{n}^{+} \mid k$, for $k+n=$ even, $n \geqslant 2$;

- from right to left as $\varepsilon$ passes through $\varepsilon_{n}^{-} / k$, for $k+n=$ even, $n \geqslant 2$.

We are now in a position to apply the results of Diekmann and van Gils [4] to obtain the (local) Hopf bifurcation of periodic solutions, as well as information about their stability near the bifurcation point. However, for this special equation we prefer a constructive proof by monotone iteration, which will provide us with a globally defined branch.

We prove the following technical result here for convenience, although it will not be needed until Section 9 .

Lemma 2.3. Suppose $0<\varepsilon \leqslant 1 / 3$. Then the characteristic equation (2.1) has at least two roots in the strip

$$
|\operatorname{Im} \mu| \leqslant \frac{\pi}{1-\varepsilon} .
$$

Proof. We first note that there can be no root with $\operatorname{Im} \mu=\pi /(1-\varepsilon)$. Indeed, if we write the characteristic equation in the form

$$
1=\frac{f^{\prime}(0)}{2 \varepsilon} \int_{1-\varepsilon}^{1+\varepsilon} e^{-\operatorname{Re} \mu \tau} e^{-i \operatorname{Im} \mu \tau} d \tau
$$

and take the imaginary part we find that necessarily

$$
0=\int_{1-\varepsilon}^{1+\varepsilon} e^{-\operatorname{Re} \mu \tau} \sin \operatorname{Im} \mu \tau d \tau,
$$

which is impossible when $\operatorname{Im} \mu=\pi /(1-\varepsilon)$ simply because then $\sin \operatorname{Im} \mu \tau$ is nonpositive for $1-\varepsilon \leqslant \tau \leqslant 1+\varepsilon$.

Next, observe that any root of (2.1) in the strip (2.3) satisfies

$$
|\operatorname{Re} \mu|<K=K\left(\varepsilon, f^{\prime}(0)\right)
$$

for some constant $K$ depending continuously on $\varepsilon>0$ and $f^{\prime}(0)<-1$. This fact is easily proved by assuming, to the contrary, the existence of sequences of numbers $\varepsilon_{n} \rightarrow \varepsilon>0$ and $f_{n}^{\prime}(0) \rightarrow f^{\prime}(0)<-1$ such that (2.1) has a corresponding sequence of roots $\mu_{n}$ in the strip (2.3) satisfying $\left|\operatorname{Re} \mu_{n}\right| \rightarrow \infty$. Taking the limit in (2.1) easily gives a contradiction.

It follows now from Rouche's Theorem that the number of roots of $(2.1)$ in the rectangle (2.3), (2.5) is independent of $\varepsilon$ and $f^{\prime}(0)$. We complete the proof of Lemma 2.3 by observing that this number is at least two, because at the particular values 
$\varepsilon=1 / 3$ and $f^{\prime}(0)=-2 \pi / 3 \sqrt{3}<-1$ we have $\varepsilon=\varepsilon^{*}$, and hence $\mu= \pm i \pi$ are two roots of $(2.1)$ in the rectangle.

\section{Monotone Iteration}

The basic space to work in will be

$$
P_{2}=\{x \in C(R) \mid x \text { is periodic with period two }\},
$$

provided with the supremum norm.

We define the following subspaces:

$$
\begin{aligned}
& P_{ \pm 1}=\left\{x \in P_{2} \mid x(t+1)= \pm x(t) \text { for all } t\right\} \\
& P^{s}=\left\{x \in P_{2} \mid x(-t)=x(t) \text { for all } t\right\} \\
& P^{a}=\left\{x \in P_{2} \mid x(-t)=-x(t) \text { for all } t\right\}
\end{aligned}
$$

and we note that $P_{2}=P_{1} \oplus P_{-1}$ and $P_{2}=P^{s} \oplus P^{a}$, the corresponding projections onto the first factor being given by $x(t) \rightarrow[x(t)+x(t+1)] / 2$ and $x(t) \rightarrow[x(t)+x(-t)] / 2$ respectively. The right hand side of $(E)$ defines a nonlinear operator $T$ as follows:

$$
(T x)(t)=\frac{1}{2 \varepsilon} \int_{1-\varepsilon}^{1+\varepsilon} f(x(t-\tau)) d \tau .
$$

When the precise value of $\varepsilon$ matters we shall write $T_{\varepsilon}$ instead of $T$.

Proposition 3.1. $\quad T: P_{2} \longrightarrow P_{2}$ leaves $P_{ \pm 1}^{a, s}$ invariant.

Proof. The invariance of $P_{ \pm 1}$ is evident. We only prove the invariance of $P^{s}$, the proof of the invariance of $P^{a}$ being similar. If $x \in P^{s}$ then

$$
\begin{aligned}
(T x)(-t) & =\frac{1}{2 \varepsilon} \int_{1-\varepsilon}^{1+\varepsilon} f(x(-t-\tau)) d \tau \\
& =\frac{1}{2 \varepsilon} \int_{-1-\varepsilon}^{-1+\varepsilon} f(x(-(t-\tau))) d \tau \\
& =\frac{1}{2 \varepsilon} \int_{-1-\varepsilon}^{-1+\varepsilon} f(x(t-\tau)) d \tau \\
& =\frac{1}{2 \varepsilon} \int_{1-\varepsilon}^{1+\varepsilon} f(x(t+2-\tau)) d \tau \\
& =\frac{1}{2 \varepsilon} \int_{1-\varepsilon}^{1+\varepsilon} f(x(t-\tau)) d \tau=(T x)(t)
\end{aligned}
$$

In $P_{-1}^{a}$ we define the closed convex cone $C^{a}$ by

$$
C^{a}=\left\{x \in P_{-1}^{a} \mid x(t) \geqslant 0 \text { for each } t \in[0,1]\right\} .
$$

Figure 6 depicts a typical element of $C^{a}$. The following observations will show that $T$ 


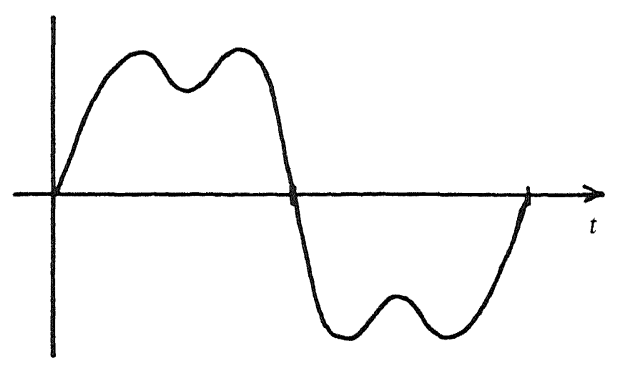

Fig. 6

maps $C^{a}$ into itself. If $x \in P_{-1}$ then the oddness of $f$ implies

$$
(T x)(t)=\frac{1}{2 \varepsilon} \int_{-\varepsilon}^{\varepsilon} g(x(t-\tau)) d \tau=\frac{1}{2 \varepsilon} \int_{t-\varepsilon}^{t+\varepsilon} g(x(\tau)) d \tau
$$

where by definition

$$
g(x)=-f(x) .
$$

If in addition $x \in P^{a}$, one can exploit the symmetry of $x$ and $g$ to reduce the interval of integration of (3.2) so that it is contained in $[0,1]$. Indeed,

$$
(T x)(t)=\frac{1}{2 \varepsilon} \int_{|t-\varepsilon|}^{1-|1-t-\varepsilon|} g(x(\tau)) d \tau, \quad \text { if } \quad t \in[0,1]
$$

for $x \in P^{a}{ }_{-1}$. This formula follows from considering separately the four cases $t-\varepsilon \leqslant 0$ or $\geqslant 0$, and $t+\varepsilon \leqslant 1$ or $\geqslant 1$. For instance if $t-\varepsilon \leqslant 0$ and $t+\varepsilon \leqslant 1$ then

$$
(T x)(t)=\frac{1}{2 \varepsilon} \int_{\varepsilon-t}^{t+\varepsilon} g(x(\tau)) d \tau
$$

whereas if $t-\varepsilon \leqslant 0$ and $t+\varepsilon \geqslant 1$

$$
(T x)(t)=\frac{1}{2 \varepsilon} \int_{\varepsilon-t}^{2-t-\varepsilon} g(x(\tau)) d \tau .
$$

We shall call this the reduction by symmetry technique. From this and the fact that $g(x) \geqslant 0$ for $x \geqslant 0$ we have the following result.

\section{Proposition 3.2. T leaves $C^{a}$ invariant.}

It turns out that still other useful properties of functions are preserved by the operator $T$. These are summarized in the next Proposition. We omit the rather tedious proof which is based on the reduction by symmetry technique.

Proposition 3.3. Let $x \in C^{a}$. If $x^{\prime}(t) \geqslant 0$ in $[-1 / 2,1 / 2]$ then $(T x)^{\prime}(t) \geqslant 0$ in $[-1 / 2,1 / 2]$. If, in addition, $x^{\prime \prime}(t) \leqslant 0$ in $[0,1]$ then $(T x)^{\prime \prime}(t) \leqslant 0$ in $[0,1]$.

We shall look for fixed points of $T$ in $C^{a}$. Note that a translate $x(t+\tau)$ of a nontrivial $x \in C^{a}$ belongs to $C^{a}$ again if and only if $\tau$ is a multiple of two, the period. 
Thus we have killed the translation invariance of $(\mathrm{E})$ by a restriction to $C^{a}$. The following a priori partial description of the form of such fixed points is needed later.

PROPOSITION 3.4. If $x \in C^{a}$ is a nontrivial fixed point of $T$, then

$$
x(t)>0 \text { for each } t \in(0,1) \text {, and } x^{\prime}(0)>0 .
$$

Proof. Suppose $x \in C^{a}$ is a nontrivial fixed point of $T$. From the reduction by symmetry technique, specifically (3.3), we see that if $x\left(t_{0}\right)>0$ for some $t_{0} \in(0,1)$ then $x(t)>0$ for all $t$ in the set

$$
I_{\varepsilon}\left(t_{0}\right)=\left\{t \in(0,1)|| t-\varepsilon\left|<t_{0}<1-\right| 1-t-\varepsilon \mid\right\} .
$$

One sees without much difficulty that $I_{\varepsilon}\left(t_{0}\right)$ is the interval

$$
I_{\varepsilon}\left(t_{0}\right)=\left(\left|t_{0}-\varepsilon\right|, 1-\left|1-t_{0}-\varepsilon\right|\right) ;
$$

therefore if $x$ is positive on some interval $\left(a_{0}, b_{0}\right) \subseteq(0,1)$ we conclude that $x(t)>0$ throughout the interval

$$
\left(a_{1}, b_{1}\right)=\bigcup_{a_{0}<t<b_{0}} I_{\varepsilon}(t) \subseteq(0,1)
$$

Clearly

$$
\begin{aligned}
& a_{1}=\min _{a_{0} \leqslant t \leqslant b_{0}}|t-\varepsilon|, \\
& b_{1}=\max _{a_{0} \leqslant t \leqslant b_{0}} 1-|1-t-\varepsilon| .
\end{aligned}
$$

Iterating this procedure shows $x(t)>0$ on $\left(a_{n}, b_{n}\right)$ where

$$
\begin{aligned}
& a_{n}=\min _{a_{n-1} \leqslant t \leqslant b_{n-1}}|t-\varepsilon|, \\
& b_{n}=\max _{a_{n-1} \leqslant t \leqslant b_{n-1}} 1-|1-t-\varepsilon| .
\end{aligned}
$$

The following facts, the proofs of which are tedious but not difficult, imply that $\left(a_{n}, b_{n}\right)=(0,1)$ for large $n$, and from this the first part of the Proposition follows. Suppose first that $\varepsilon \leqslant 1 / 2$. Then

(i) if $a_{n-1}<b_{n-1}<\varepsilon$, then $a_{n}<\varepsilon<b_{n}$;

(ii) if $1-\varepsilon<a_{n-1}<b_{n-1}$, then $a_{n}<1-\varepsilon<b_{n}$; and

(iii) if neither (i) nor (ii) holds then

$$
\left(a_{n}, b_{n}\right)=\left(a_{n-1}-\varepsilon, b_{n-1}+\varepsilon\right) \cap(0,1) .
$$

We see that after one iteration we are in, and remain in, case (iii), and that $\left(a_{n}, b_{n}\right)=$ $(0,1)$ for large $n$.

If on the other hand $\varepsilon>1 / 2$, then

(iv) if $a_{n-1}<b_{n-1}<1-\varepsilon$, then $a_{n}<\varepsilon<b_{n}$;

(v) if $\varepsilon<a_{n-1}<b_{n-1}$, then $a_{n}<1-\varepsilon<b_{n}$; and

(vi) if neither (iv) nor (v) holds then 


$$
\left(a_{n}, b_{n}\right)=\left(\varepsilon-b_{n-1}, 2-a_{n-1}-\varepsilon\right) \cap(0,1) .
$$

Again, after the first iteration we are in case (vi). From there it is not difficult to see that the interval $\left(a_{n}, b_{n}\right)$ increases its length by at least $1-\varepsilon$ per iteration, until $\left(a_{n}, b_{n}\right)=(0,1)$ as required.

This completes the proof that $x(t)>0$ in $(0,1)$. To finish the proof of the Proposition we simply note that from the differentiated form (1.5) of (E), and the symmetry conditions, we have

$$
x^{\prime}(0)=\frac{1}{\varepsilon} g(x(\varepsilon))>0 .
$$

The linearization at zero of the restriction of $T$ to $P_{-1}$ is given by $g^{\prime}(0) M$, where we define

$$
(M x)(t)=\frac{1}{2 \varepsilon} \int_{-\varepsilon}^{\varepsilon} x(t-\tau) d \tau .
$$

In the following we shall use $\leqslant$ in the sense of the cone $C^{a}$; that is, $x \leqslant y$ if and only if $y-x \in C^{a}$.

\section{PROPOSITION 3.5.}

(i) If $\|x\| \leqslant 1$ then $\|T x\| \leqslant 1$.

(ii) $T$ is order preserving: if $x, y \in C^{a}$ with $x \leqslant y$, then $T x \leqslant T y$.

(iii) Let $\phi \in C^{a}$ be defined by $\phi(t)=\sin \pi t$. Then for each $\delta>0$ there exists $\mu<1$ such that

$$
T(\delta \phi) \leqslant \mu g^{\prime}(0) M(\delta \phi)=\mu g^{\prime}(0)\left(\frac{\sin \pi \varepsilon}{\pi \varepsilon}\right) \delta \phi .
$$

(iv) For each $\gamma \in\left(0, g^{\prime}(0)\right)$ define $\delta$ to be the unique positive root of the equation $g(x)=\gamma x$. Then

$$
T x \geqslant \gamma M x \text { for each } x \in C^{a} \text { with }\|x\| \leqslant \delta .
$$

Proof. The properties of $g(x)=-f(x)$ and the reduction by symmetry technique imply (i), (ii) and (iv) at once. The proof of (iii) follows these lines, but requires a bit more work to obtain the strict inequality $\mu<1$. It is easy to show the strict inequality

$$
T(\delta \phi)(t)<g^{\prime}(0)\left(\frac{\sin \pi \varepsilon}{\pi \varepsilon}\right) \delta \phi(t)
$$

at each $t$ in the open interval $(0,1)$. The existence of $\mu<1$ thus follows by considering the limit of the ratios

$$
\lim _{t \rightarrow 0} \frac{T(\delta \phi)(t)}{\delta \phi(t)}=\lim _{t \rightarrow 1} \frac{T(\delta \phi)(t)}{\delta \phi(t)}
$$


whose value, by l'Hôpital's rule, is

$$
\frac{T(\delta \phi)^{\prime}(0)}{\delta \phi^{\prime}(0)}=\frac{g(\delta \phi(\varepsilon))}{\varepsilon \delta \phi^{\prime}(0)}<g^{\prime}(0) \frac{\delta \phi(\varepsilon)}{\varepsilon \delta \phi^{\prime}(0)}=g^{\prime}(0)\left(\frac{\sin \pi \varepsilon}{\pi \varepsilon}\right) .
$$

This implies the result.

THEOREM 3.6.

(i) $T$ has no nontrivial fixed point in $C^{a}$ if $\sin \pi \varepsilon \leqslant \pi \varepsilon / g^{\prime}(0)$.

(ii) If, on the other hand, $\sin \pi \varepsilon>\pi \varepsilon / g^{\prime}(0)$ then $T$ has a unique nontrivial fixed point $x_{\varepsilon}$ in $C^{a}$. Moreover, $\left|x_{\varepsilon}(t)\right|<1$ for all $t, x_{\varepsilon}^{\prime}(t)>0$ if $t \in(-1 / 2,1 / 2)$, and $x_{\varepsilon}^{\prime \prime}(t)<0$ if $t \in(0,1)$.

Proof. The existence or nonexistence of a fixed point $x_{\varepsilon}$ is a standard application of the theory of concave operators presented in Krasnosel'skii's book [16]. For completeness we give a brief sketch.

Suppose $\sin \pi \varepsilon \leqslant \pi \varepsilon / g^{\prime}(0)$, and that $x \in C^{a}$ is a nontrivial fixed point of $T$. We have $x \leqslant \delta \phi$ for sufficiently large $\delta$ (with $\phi(t)=\sin \pi t$ as before) because $x^{\prime}(0)=$ $(1 / \varepsilon) g(x(\varepsilon))=-x^{\prime}(1)$ is finite. Set $\delta^{*}=\inf \{\delta \mid x \leqslant \delta \phi\}$, and note $\delta^{*}>0$. From (ii) and (iii) of Proposition 3.5, we have for some $\mu<1$ that

$$
x=T x \leqslant T\left(\delta^{*} \phi\right) \leqslant \mu g^{\prime}(0)\left(\frac{\sin \pi \varepsilon}{\pi \varepsilon}\right) \delta^{*} \phi \leqslant \mu \delta^{*} \phi .
$$

This contradicts the definition of $\delta^{*}$.

If on the other hand $\sin \pi \varepsilon>\pi \varepsilon / g^{\prime}(0)$ then one can choose $\gamma \in\left(0, g^{\prime}(0)\right)$ and $\delta$ as in (iv) of Proposition 3.5, so that $\delta<1$ and

$$
T(\delta \phi) \geqslant \gamma M(\delta \phi)=\gamma\left(\frac{\sin \pi \varepsilon}{\pi \varepsilon}\right) \delta \phi \geqslant \delta \phi .
$$

As a consequence the sequence $T^{n}(\delta \phi)$ increases monotonically; it is equicontinuous and is bounded above by one, so must converge to a limit $x_{\varepsilon} \in C^{a}$, which is necessarily a fixed point of $T$. Of course this fixed point is also uniformly bounded by one.

Let $y$ be any other nontrivial fixed point in $C^{a}$ and define

$$
h(t)= \begin{cases}\frac{x_{\varepsilon}(t)}{y(t)}, & 0<t<1 \\ \frac{x_{\varepsilon}^{\prime}(0)}{y^{\prime}(0)}, & t=0,1 .\end{cases}
$$

By Proposition 3.4 the function $h$ is well-defined, positive, and continuous in $[0,1]$. Let $\alpha=\inf \{h(t) \mid 0 \leqslant t \leqslant 1\}$ and assume $\alpha<1$. The strict inequality

$$
g(\theta x)>\theta g(x) \quad \text { if } \quad x \in(0, \infty) \text { and } \theta \in(0,1)
$$

(which follows from $\left(H_{4}\right)$ ), and the fact that $y(\varepsilon)>0$ (by Proposition 3.4), implies that 


$$
x_{\varepsilon}^{\prime}(0)=\frac{1}{\varepsilon} g\left(x_{\varepsilon}(\varepsilon)\right) \geqslant \frac{1}{\varepsilon} g(\alpha y(\varepsilon))>\frac{\alpha}{\varepsilon} g(y(\varepsilon))=\alpha y^{\prime}(0) .
$$

Consequently, $h$ cannot attain its infimum at $t=0$ or $t=1$. Similarly, if $t \in(0,1)$ we have

$$
\begin{aligned}
x_{\varepsilon}(t) & =\frac{1}{2 \varepsilon} \int_{|t-\varepsilon|}^{1-|1-t-\varepsilon|} g\left(x_{\varepsilon}(\tau)\right) d \tau \geqslant \frac{1}{2 \varepsilon} \int_{|t-\varepsilon|}^{1-|1-t-\varepsilon|} g(\alpha y(\tau)) d \tau \\
& >\frac{\alpha}{2 \varepsilon} \int_{|t-\varepsilon|}^{1-|1-t-\varepsilon|} g(y(\tau)) d \tau=\alpha y(t)
\end{aligned}
$$

so the infimum is not attained in $(0,1)$. Thus $\alpha<1$ is impossible, so $\alpha \geqslant 1$. But reversing the roles of $x_{\varepsilon}$ and $y$ and considering $1 / h(t)=y(t) / x_{\varepsilon}(t)$ shows that $\alpha \leqslant 1$. Hence $h(t) \equiv 1$, and $y=x_{\varepsilon}$. This proves the existence of a unique fixed point. We shall continue to denote this fixed point by $x_{\varepsilon}$.

The fact that $\delta \phi(t)=\delta \sin \pi t$ is nondecreasing in $[-1 / 2,1 / 2]$ and concave on $[0,1]$ implies, by Proposition 3.3, that the limit $T^{n}(\delta \phi) \rightarrow x_{\varepsilon}$ also has these properties. To prove the strict inequality $x_{\varepsilon}^{\prime}(t)>0$ in $(-1 / 2,1 / 2)$, we first note that $x_{\varepsilon}^{\prime}(0)>0$, and let $t_{0} \in(0,1 / 2]$ be the first positive value at which $x_{\varepsilon}^{\prime}$ vanishes. We wish to show that $t_{0}=1 / 2$. The symmetry of $x_{\varepsilon}$ and the fact that it is concave and nondecreasing in $[0,1 / 2]$ imply that

$$
\begin{cases}x_{\varepsilon}(t)=\left\|x_{\varepsilon}\right\| & \text { if } t \in\left[t_{0}, 1-t_{0}\right], \\ x_{\varepsilon}(t)=-\left\|x_{\varepsilon}\right\| & \text { if } t \in\left[-1+t_{0},-t_{0}\right], \text { and } \\ -\left\|x_{\varepsilon}\right\|<x_{\varepsilon}(t)<\left\|x_{\varepsilon}\right\| & \text { if } t \in\left(-t_{0}, t_{0}\right) .\end{cases}
$$

Also note that

$$
x_{\varepsilon} \text { is one-to-one on }\left(-t_{0}, t_{0}\right) \text {. }
$$

From (1.5) we have $x_{\varepsilon}^{\prime}(t)=\left[g\left(x_{\varepsilon}(t+\varepsilon)\right)-g\left(x_{\varepsilon}(t-\varepsilon)\right)\right] / 2 \varepsilon$, and as $x_{\varepsilon}^{\prime}\left(t_{0}\right)=0$ it follows that

$$
x_{\varepsilon}\left(t_{0}-\varepsilon\right)=x_{\varepsilon}\left(t_{0}+\varepsilon\right)=x_{\varepsilon}\left(1-t_{0}-\varepsilon\right) .
$$

The inequalities $t_{0}-1<t_{0}-\varepsilon<t_{0}$ and $-t_{0}<1-t_{0}-\varepsilon<1-t_{0}$, together with (3.4) and (3.6), imply that

$$
t_{0}-\varepsilon, \quad 1-t_{0}-\varepsilon \in\left(-t_{0}, t_{0}\right) .
$$

And (3.5), (3.6), and (3.7) imply that $t_{0}-\varepsilon=1-t_{0}-\varepsilon$, hence $t_{0}=1 / 2$ as desired. This completes the proof that $x_{\varepsilon}^{\prime}(t)>0$ for each $t \in(-1 / 2,1 / 2)$.

As noted before, $\left|x_{\varepsilon}(t)\right| \leqslant 1$ for all $t$. This inequality is strict if $t \in[0,1]-\{1 / 2\}$ because $x_{\varepsilon}^{\prime}(t) \neq 0$ there. At the maximum $t=1 / 2$ we also have a strict inequality as the integrand in 


$$
x_{\varepsilon}\left(\frac{1}{2}\right)=\frac{1}{2 \varepsilon} \int_{-\varepsilon}^{\varepsilon} g\left(x_{\varepsilon}\left(\frac{1}{2}-\tau\right)\right) d \tau
$$

is strictly less than one if $\tau \neq 0$. Thus $\left|x_{\varepsilon}^{*}(t)\right|<1$ for all $t$.

To show that $x_{\varepsilon}^{\prime \prime}(t)<0$ in $(0,1)$, first note that the (not necessarily strict) concavity of $x_{\varepsilon}$ in this interval, together with the fact that $g^{\prime}(x)>0$ is a strictly decreasing function of $x$, implies that $g^{\prime}(x(t)) x^{\prime}(t)$ is a strictly decreasing function of $t$ in $(0,1)$. This and the formula

$$
x_{\varepsilon}^{\prime \prime}(t)=\frac{1}{2 \varepsilon}\left[g^{\prime}\left(x_{\varepsilon}(t+\varepsilon)\right) x_{\varepsilon}^{\prime}(t+\varepsilon)-g^{\prime}\left(x_{\varepsilon}(t-\varepsilon)\right) x_{\varepsilon}^{\prime}(t-\varepsilon)\right]
$$

obtained from (1.5) easily implies that $x_{\varepsilon}^{\prime \prime}(t)<0$ for each $t \in(0,1)$.

Proposition 2.1 and Theorem 3.6 immediately imply the following result.

COROLlaRy 3.7. $T$ has a unique nontrivial fixed point $x_{\varepsilon} \in C^{a}$ for each $0<\varepsilon<\varepsilon^{*}$.

It is easy to verify that if $x(t)$ is any two-periodic solution of (E) for some $\varepsilon>0$, and if $k$ is odd, then $y(t)=x(k t)$ is a $2 / k$-periodic solution of $(\mathrm{E})$ but with parameter $\varepsilon / k$. Applying this rescaling procedure to the solution $x_{\varepsilon}$ obtained above thus generates other periodic solutions.

THEOREM 3.8. If $k$ is odd and $\varepsilon<\varepsilon^{*} / k$, then the function $y(t)=x_{k \varepsilon}(k t)$ is a solution of $(\mathrm{E})$ of period $2 / k$.

With further effort it is possible to obtain still other periodic solutions of $(E)$ for $\varepsilon$ lying in the intervals $\left(\varepsilon_{n}^{-} / k, \varepsilon_{n}^{+} / k\right)$ between the Hopf bifurcation points. Consider first the case when $n \geqslant 3$ is odd. We first obtain a solution for $\varepsilon_{n}^{-}<\varepsilon<\varepsilon_{n}^{+}$, and then rescale. Note that even though we consider values of $\varepsilon$ greater than one, our arguments are valid.

LEMMA 3.9. Let $n \geqslant 3$ be odd and assume that $\varepsilon_{n}^{ \pm}$exist. If $\varepsilon_{n}^{-}<\varepsilon<\varepsilon_{n}^{+}$then $T_{\varepsilon}$ has a unique nontrivial fixed point $x_{\varepsilon}$ in $C^{a}$.

THEOREM 3.10. If $n \geqslant 3$ and $k$ are odd, and $\varepsilon_{n}^{-} / k<\varepsilon<\varepsilon_{n}^{+} / k$, then $y(t)=x_{k \varepsilon}(k t)$ is a $2 / k$-periodic solution of $(\mathrm{E})$.

We prove only Lemma 3.9, as the proof of Theorem 3.10 is straightforward. Again note that the range of $\varepsilon$ considered in Lemma 3.9 is $\left(\varepsilon_{n}^{-}, \varepsilon_{n}^{+}\right) \subseteq(n-1, n)$, so the fixed point $x_{\varepsilon}$ is different from the one obtained above in Corollary 3.7.

Proof of Lemma 3.9. Note that $n-1<\varepsilon<n$, and set $\delta=\varepsilon-(n-1)$. Let $x \in P_{-1}^{a}$; then using the fact that $n-1$ is even and that the mean value of $f(x(t))$ is zero yields

$$
\left(T_{\varepsilon} x\right)(t)=\frac{1}{2 \varepsilon} \int_{1-\varepsilon}^{1+\varepsilon} f(x(t-\tau)) d \tau=\frac{1}{2 \varepsilon} \int_{1-\delta}^{1+\delta} f(x(t-\tau)) d \tau=\frac{\delta}{\varepsilon}\left(T_{\delta} x\right)(t) .
$$


Thus we seek a solution $x \in C^{a}$ to the problem $x=(\delta / \varepsilon) T_{\delta} x$. Exactly as in the proof of Theorem 3.6, a necessary and sufficient condition for a nontrivial solution to exist is $(\delta / \varepsilon)(\sin \pi \delta)>\pi \delta / g^{\prime}(0)$, or equivalently, $\sin \pi \varepsilon>\pi \varepsilon / g^{\prime}(0)$. This holds if and only if $\varepsilon_{n}^{-}<\varepsilon<\varepsilon_{n}^{+}$. The solution $x_{\varepsilon}$ moreover is unique and can be obtained by monotone iteration.

The case when $n$ is even requires some slight modifications due to the fact that $k$ also must be even. Note in particular that the fixed point $y_{\varepsilon}$ does not lie in the symmetry class $P_{-1}$, so we must use the original definition (3.1) of $T_{\varepsilon}$ rather than the formula (3.2).

LEMMA 3.11. Let $n$ be even and assume that $\varepsilon_{n}^{ \pm}$exist. If $\varepsilon_{n}^{-} / 2<\varepsilon<\varepsilon_{n}^{+} / 2$ then $T_{\varepsilon}$ (given by (3.1)) has a unique nontrivial fixed point $y_{\varepsilon}$ of the form $y_{\varepsilon}(t)=x_{2 \varepsilon}(2 t)$, where $x_{2 \varepsilon} \in C^{a}$.

THEOREM 3.12. If $n$ and $k$ are even, and $\varepsilon_{n}^{-} \mid k<\varepsilon<\varepsilon_{n}^{+} / k$, then $y(t)=x_{k \varepsilon}(k t)$ is a $2 / k$-periodic solution of $(\mathrm{E})$.

We omit the proofs of these results, as they are similar to those given previously. We note that the two-periodia functions $x_{\varepsilon}$ and $x_{2 \varepsilon}$ obtained in Lemmas 3.9 and 3.11 satisfy the monotonicity and convexity conditions as in the statement of Theorem 3.6, as well as the strict bound $\left\|x_{\varepsilon}\right\|,\left\|x_{2 \varepsilon}\right\|<1$.

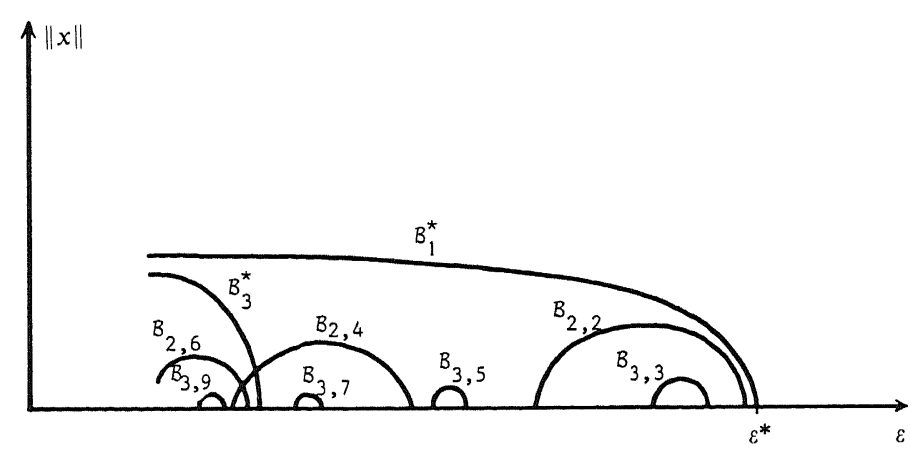

Fig. 7

Note that the order in which the Hopf bifurcations occur as $\varepsilon$ decreases is not necessarily the same as the order of the periods of the associated periodic solutions. Although the bifurcation points $\varepsilon^{*}>\varepsilon^{*} / 3>\varepsilon^{*} / 5>\cdots$ correspond to periods $2>2 / 3>2 / 5>\cdots$ with the same ordering, it is also possible to have, for example, for a suitable nonlinearity $f$, bifurcation points $\varepsilon_{3}^{+} / 5>\varepsilon^{*} / 3$ with corresponding periods $2 / 5<2 / 3$. Note however that $\varepsilon^{*}>\varepsilon_{2}^{+} / 2>\varepsilon_{3}^{+} / 3>\cdots$ which implies that as $\varepsilon$ decreases in the interval $(0,1)$ the first Hopf bifurcation encountered is at $\varepsilon=\varepsilon^{*}$; see Figs. 5 and 7 and Table 1.

Table 1 gives the values of the parameter $\varepsilon$ at the various Hopf bifurcations in the interval $.25<\varepsilon<1$ for the case $f^{\prime}(0)=-8$. Figure 7 depicts the corresponding 
Table 1. The Hopf bifurcation points $\varepsilon \in \varepsilon^{*} / k$ and $\varepsilon=\varepsilon_{n}^{ \pm} / k$ in the range $.25<\varepsilon<1$ for $f^{\prime}(0)=-8$.

The resulting global branches of solutions are shown in Fig. 7.

\begin{tabular}{ccc}
\hline Bifurcation Point & Value of $\varepsilon$ & Period of Solution \\
\hline$\varepsilon^{*}$ & .887 & 2 \\
$\varepsilon_{2}^{+} / 2$ & .879 & 1 \\
$\varepsilon_{3}^{+} / 3$ & .844 & $2 / 3$ \\
$\varepsilon_{3}^{-} / 3$ & .796 & $2 / 3$ \\
$\varepsilon_{2}^{-} / 2$ & .575 & 1 \\
$\varepsilon_{3}^{+} / 5$ & .507 & $2 / 5$ \\
$\varepsilon_{3}^{-} / 5$ & .477 & $2 / 5$ \\
$\varepsilon_{2}^{+} / 4$ & .439 & $1 / 2$ \\
$\varepsilon_{3}^{+} / 7$ & .362 & $2 / 7$ \\
$\varepsilon_{3}^{-} / 7$ & .341 & $2 / 7$ \\
$\varepsilon^{*} / 3$ & .296 & $2 / 3$ \\
$\varepsilon_{2}^{+} / 6$ & .293 & $1 / 3$ \\
$\varepsilon_{2}^{-} / 4$ & .287 & $1 / 2$ \\
$\varepsilon_{3}^{+} / 9$ & .281 & $2 / 9$ \\
$\varepsilon_{3}^{-} / 9$ & .265 & $2 / 9$ \\
\hline
\end{tabular}

global branches $\mathscr{B}_{k}^{*}$ and $\mathscr{B}_{n, k}$ emanating from $\varepsilon^{*} / k$ and $\varepsilon_{n}^{ \pm} / k$ respectively whose existence is proved above. (Of course, as $\varepsilon \downarrow 0$ there is an infinite sequence of such bifurcations, clustering at zero.) We will prove in Section 7 that the solutions on these branches vary smoothly in $\varepsilon$ as shown; but as noted earlier, we cannot at this point rule out the possibility of secondary bifurcations, or the existence of other periodic solutions not arising from the Hopf bifurcations.

We contrast the situation here with that for certain scalar differential-delay equations such as $x^{\prime}(t)=\alpha f(x(t-1))$ and $\varepsilon x^{\prime}(t)=-x(t)+f(x(t-1))$ in which the periods of the bifurcating solutions are ordered monotonically with respect to the bifurcation parameter. See [2, 17, 19, 20, 23, 26].

\section{Monotonicity with Respect to $\varepsilon$}

For the remainder of this paper, unless otherwise stated, $x_{\varepsilon}$ denotes the unique nontrivial fixed point of $T$ in $C^{a}$ for $\varepsilon<\varepsilon^{*}$. In order to show that $x_{\varepsilon}$ depends monotonically (in the sense of $C^{a}$ ) on $\varepsilon$ the following elementary Lemmas are useful. We omit the proofs of the first two, as they are quite simple.

LEMMA 4.1. If the function $h$ is concave on $[a, b]$ then

$$
\int_{a}^{b} h(\tau) d \tau \geqslant \frac{b-a}{2}[h(b)+h(a)],
$$

with strict inequality unless $h$ is linear in $[a, b]$.

LEMMA 4.2. Let the function $h$ be concave on $[0,1]$ and satisfy $h(0)=0$. For 
arbitrary $\tau \in[0,1]$ define $H(t)=\tau h(t)-\operatorname{th}(\tau)$. Then $H(t) \leqslant 0$ if $t \in[\tau, 1]$.

LEMMA 4.3. Let $x$ be a fixed element of $C^{a}$ which is strictly concave on $(0,1)$. Then

$$
\frac{d}{d \varepsilon}\left(T_{\varepsilon} x\right)(t)<0 \quad \text { if } \quad t \in(0,1)
$$

Proof. As usual we employ the reduction by symmetry technique, restricting our attention to $t \in(0,1 / 2]$. Four cases arise.

(i) $t-\varepsilon \leqslant 0$ and $t+\varepsilon \leqslant 1$ :

$$
\begin{aligned}
\frac{d}{d \varepsilon}\left(T_{\varepsilon} x\right)(t) & =-\frac{1}{2 \varepsilon^{2}} \int_{\varepsilon-t}^{t+\varepsilon} g(x(\tau)) d \tau+\frac{1}{2 \varepsilon}[g(x(t+\varepsilon))-g(x(\varepsilon-t))] \\
& <\frac{1}{2 \varepsilon^{2}}[(\varepsilon-t) g(x(\varepsilon+t))-(\varepsilon+t) g(x(\varepsilon-t))] \leqslant 0 .
\end{aligned}
$$

Here we used Lemma 4.1 in the first inequality and Lemma 4.2 in the second.

(ii) $t-\varepsilon \leqslant 0$ and $t+\varepsilon \geqslant 1$ :

$$
\frac{d}{d \varepsilon}\left(T_{\varepsilon} x\right)(t)=-\frac{1}{2 \varepsilon^{2}} \int_{\varepsilon-t}^{2-t-\varepsilon} g(x(\tau)) d \tau-\frac{1}{2 \varepsilon} g(x(2-t-\varepsilon))-\frac{1}{2 \varepsilon} g(x(\varepsilon-t))<0 .
$$

(iii) $t-\varepsilon \geqslant 0$ and $t+\varepsilon \leqslant 1$ :

$$
\frac{d}{d \varepsilon}\left(T_{\varepsilon} x\right)(t)=-\frac{1}{2 \varepsilon^{2}} \int_{t-\varepsilon}^{t+\varepsilon} g(x(\tau)) d \tau+\frac{1}{2 \varepsilon}[g(x(t+\varepsilon))+g(x(t-\varepsilon))]<0 .
$$

(iv) $t-\varepsilon>0$ and $t+\varepsilon>1$ is impossible for $t \in(0,1 / 2]$.

THEOREM 4.4. $\varepsilon_{2}<\varepsilon_{1}$ implies $x_{\varepsilon_{2}}(t)>x_{\varepsilon_{1}}(t)$ for each $t \in(0,1)$.

Proof. Lemma 4.3 implies that $T_{\varepsilon_{1}} x_{\varepsilon_{1}}(t)<T_{\varepsilon_{2}} x_{\varepsilon_{1}}(t)$ if $t \in(0,1)$, and therefore $x_{\varepsilon_{1}}(t)<T_{\varepsilon_{2}} x_{\varepsilon_{1}}(t)$. So we can use $x_{\varepsilon_{1}}$ as the starting point for a monotone iteration with $T_{\varepsilon_{2}}$. Since the fixed point is unique in $C^{a}$, the limit of the sequence $T_{\varepsilon_{2}}^{n} x_{\varepsilon_{1}}$ is necessarily $x_{\varepsilon_{2}}$.

COROLlaRY 4.5. $\quad x_{\varepsilon}(t) \uparrow 1$ as $\varepsilon \downarrow 0$ uniformly on compact subsets of $(0,1)$.

Proof. The monotonicity implies that $x_{\varepsilon}(t) \uparrow x_{0}(t) \leqslant 1$ as $\varepsilon \downarrow 0$. By taking limits in the equation we find $x_{0}(t)=g\left(x_{0}(t)\right)$ and therefore $x_{0}(t)=1$ if $t \in(0,1)$. Finally, Dini's Theorem implies that the convergence is uniform on compact subsets of $(0,1)$.

We can reformulate Corollary 4.5 by saying that $x_{\varepsilon}$ converges to the square wave $\operatorname{sqw}(t)$, defined in the Introduction, as $\varepsilon \downarrow 0$.

Since the $2 / k$-periodic solutions (for $k$ odd) given by Theorem 3.8 are generated by $x_{\varepsilon}$ through a time-scaling with a factor $k$, we know that these converge to scaled versions of the square-wave as $\varepsilon \downarrow 0$. Finally, we observe that the periodic solutions in the windows $\left(\varepsilon_{n}^{-}, \varepsilon_{n}^{+}\right)$are not necessarily monotone with respect to $\varepsilon$ since they are 
found as solutions of $x=\alpha T x$ with some $\alpha=\alpha(\varepsilon)<1$.

\section{The Transition Layer}

Remarkably, the transformation $y(t)=x(\varepsilon t)$ leads to the $\varepsilon$-independent equation

$$
y(t)=\frac{1}{2} \int_{-1}^{1} g(y(t-\tau)) d \tau
$$

when $x \in P_{-1}$. In particular, if we take $x=x_{\varepsilon}$ then the corresponding solutions $y_{\varepsilon}$ of (TLE) are periodic with period $2 / \varepsilon$. Moreover, each $y_{\varepsilon}$ is increasing on $(-1 / 2 \varepsilon, 1 / 2 \varepsilon)$, concave on $(0,1 / \varepsilon)$, bounded independent of $\varepsilon$, and satisfies $y_{\varepsilon}(-t)=-y_{\varepsilon}(t)$ for all $t$ and $y_{\varepsilon}(1 / 2 \varepsilon) \uparrow 1$ as $\varepsilon \downarrow 0$. By the Arzela-Ascoli Theorem, the limit set of the family $\left\{y_{\varepsilon}\right\}$ is non-empty. Each element $y_{0}$ of this limit set is a bounded, nondecreasing, antisymmetric solution of (TLE) which is concave on $(0, \infty)$. We want to exclude the possibility that $y \equiv 0$ belongs to the limit set; this can only happen if for some sequence $\varepsilon_{n} \downarrow 0$ we have $y_{\varepsilon_{n}}(t) \rightarrow 0$ uniformly on compact sets. Assume this occurs, and define $t_{n}$ by $y_{\varepsilon_{n}}\left(t_{n}\right)=1 / 2$. Necessarily $t_{n} \rightarrow \infty$. The sequence of functions $y_{\varepsilon_{n}}\left(t+t_{n}\right)$ also has a non-empty limit set, each element of which is a solution $y$ of (TLE) which is concave and bounded on $(-\infty, \infty)$, and satisfies $y(0)=1 / 2$. These conditions imply that $y$ is the constant function $1 / 2$; however, this does not satisfy (TLE). From this contradiction we conclude that the zero function does not belong to the limit set of $\left\{y_{\varepsilon}\right\}$, and hence the limit set of $\left\{y_{\varepsilon}\right\}$ contains a nontrivial element. We see from the following Theorem that this element $y_{0}$ is unique and satisfies some additional properties. Figure 8 depicts the graph of $y_{0}$.

THEOREM 5.1. There is a unique nontrivial solution $y_{0}$ of (TLE) among the class of bounded, nondecreasing, anti-symmetric functions. This solution satisfies $y_{0}(-\infty)=$ -1 and $y_{0}(\infty)=1$. In addition, $y_{0}^{\prime}(t)>0$ for each real $t$, and $y_{0}^{\prime \prime}(t)<0$ if $t>0$. Finally, we have

$$
x_{\varepsilon}(\varepsilon t) \longrightarrow y_{0}(t) \quad \text { as } \quad \varepsilon \downarrow 0
$$

uniformly for $t$ in compact sets.



Fig. 8 
Proof. The existence of a nontrivial, bounded, nondecreasing anti-symmetric solution $y_{0}$ has already been established. In addition, we have noted that $y_{0}^{\prime}(t) \geqslant 0$ for all $t$ and $y_{0}^{\prime \prime}(t) \leqslant 0$ if $t>0$. The proofs that these inequalities are actually strict are simple arguments similar to those in the proof of Theorem 3.6, so we omit them. Also, the limits of $y_{0}(t)$ as $t \rightarrow \pm \infty$ are easily seen to be the two nontrivial fixed points \pm 1 of $g$, as claimed.

Let $y_{1}$ be any other nontrivial, bounded, nondecreasing, anti-symmetric solution of (TLE) and define

$$
h(t)= \begin{cases}\frac{y_{0}(t)}{y_{1}(t)}, & t \neq 0, \\ \frac{y_{0}^{\prime}(0)}{y_{1}^{\prime}(0)}, & t=0 .\end{cases}
$$

Observe that $y_{1}$, like $y_{0}$, satisfies $y_{1}^{\prime}(t)>0$ for all $t$, and that $\lim _{t \rightarrow \pm \infty} y_{1}(t)= \pm 1$. Thus $h$ is positive and continuous, and $\lim _{t \rightarrow \pm \infty} h(t)=1$. Let $\alpha=\inf \{h(t) \mid t \in(-\infty, \infty)\}$ and assume without loss of generality that $\alpha<1$. Necessarily then $h\left(t_{0}\right)=\alpha$ for some $t_{0} \in[0, \infty)$. Exactly as in the proof of Theorem 3.6 we obtain a contradiction.

Finally, we may conclude (5.1) from the fact that the sequence $\varepsilon_{n}$ used to obtain the unique $y_{0}$ is arbitrary.

\section{The Characteristic Multiplier $\alpha=1$}

In [4] a dynamical system approach for Volterra integral equations of convolution type is developed. Although the Floquet theory for periodic solutions is not treated, the similarities with the theory for functional differential equations, as described in Hale [9], is clear. Associated with the two-periodic solution $x_{\varepsilon}$ is the nonautonomous linear variational equation

$$
z(t)=\frac{1}{2 \varepsilon} \int_{1-\varepsilon}^{1+\varepsilon} f^{\prime}\left(x_{\varepsilon}(t-\tau)\right) z(t-\tau) d \tau .
$$

A complex number $\mu$ is a characteristic exponent in case (LE) has a non-trivial solution (or eigenfunction)

$$
z(t)=e^{\mu t} p(t), \quad \text { where } \quad p(t+2)=p(t) .
$$

The values $\alpha=e^{2 \mu}$ are called the characteristic multipliers. Corresponding to the solution

$$
z(t)=x_{\varepsilon}^{\prime}(t)
$$

is the trivial multiplier $e^{2 \mu}=1$. In this Section we shall derive some preliminary results about the multiplier $\alpha=1$, with an eye to proving in Section 9 that it is a simple multiplier. This, together with further results derived from the theory of slowly oscillating solutions developed in Section 8 , will eventually lead to a stability proof 
for $x_{\varepsilon}$. Indeed, to prove asymptotic stability with asymptotic phase it is enough to show that $\alpha=1$ is a simple multiplier and $|\alpha|<1$ for all other multipliers. And if $|\alpha|>1$ for some multiplier then $x_{\varepsilon}$ will be unstable.

As with the Floquet theory for ordinary differential equations, a multiplier $\alpha=e^{2 \mu}$ can fail to be simple in two different ways. There may exist more than one linearly independent eigenfunction, say $z(t)=e^{\mu t} p_{n}(t), n=1,2$. Or there may exist an eigenfunction (6.1) together with some exponential-polynomial solutions of (LE); in particular there must exist a solution $z$ of the form

$$
z(t)=e^{\mu t}[t p(t)+v(t)], \quad \text { where } \quad v(t+2)=v(t) .
$$

One result of this Section is that there are no solutions of the form (6.2) with $\mu=0$ and $p(t)=x_{\varepsilon}^{\prime}(t)$. We shall also show that $x_{\varepsilon}^{\prime}$ is the only solution (up to scalar multiple) of (LE) in the symmetry class $P_{-1}$. Hence, as the kernel $f^{\prime}\left(x_{\varepsilon}(t)\right)$ has period one, the only way $\alpha=1$ can fail to be simple is if (LE) has a nontrivial solution $z \in P_{1}$. The possibility of such a solution will then be ruled out in Section 9 .

Let $L: P_{2} \rightarrow P_{2}$ be the linear operator

$$
(L z)(t)=\frac{1}{2 \varepsilon} \int_{1-\varepsilon}^{1+\varepsilon} f^{\prime}\left(x_{\varepsilon}(t-\tau)\right) z(t-\tau) d \tau .
$$

Clearly $L$ is a compact operator leaving the subspaces $P_{ \pm 1}^{a, s}$ and the cones $C^{a}$ and

$$
C^{s}=\left\{z \in P_{-1}^{s} \mid z(t) \geqslant 0 \text { for each } t \in\left[-\frac{1}{2}, \frac{1}{2}\right]\right\}
$$

invariant. Also $L x_{\varepsilon}^{\prime}=x_{\varepsilon}^{\prime} \in C^{s}$. (Caution: Except for $\alpha=1$, the eigenvalues of $L$ bear no relation to the characteristic multipliers of Floquet theory.)

We recall some facts from the theory of positive linear operators. Let $A$ be a bounded linear operator leaving invariant a cone $C$ in a Banach space $X$ and let $u_{0}$ denote some fixed non-zero element of $C$. $A$ is called $u_{0}$-positive if for every $z \in C \backslash\{0\}$ one can find an integer $n$ and positive numbers $\alpha$ and $\beta$ such that $\alpha u_{0} \leqslant A^{n} z \leqslant \beta u_{0}$. The cone $C$ is called reproducing if $X$ is spanned by $C$, i.e., $X=C-C$.

THEOREM 6.1. Let $C$ be reproducing and let $A$ be a positive compact and $u_{0}$ positive operator for some $u_{0} \in C \backslash\{0\}$. Then

(i) there exists $z_{0} \in C \backslash\{0\}$ such that $A z_{0}=\lambda_{0} z_{0}$, where $\lambda_{0}=$ spectral radius of $A$, is an algebraically simple eigenvalue;

(ii) $z_{0}$ is the only eigenvector of $A$ in $C$;

(iii) there exists a strictly positive $F_{0} \in C^{*} \backslash\{0\}$ such that $A^{*} F_{0}=\lambda_{0} F_{0}$; and

(iv) all other eigenvalues $\lambda$ of $A$ satisfy $|\lambda|<\lambda_{0}$.

For the proof of this well-known Krein-Rutman type theorem we refer to [16]. We may apply this result to the operator $L$ restricted to $P_{-1}^{a, s}$.

LEMMA 6.2. Let $P_{-1}^{a}$ and $P_{-1}^{s}$ be ordered by the reproducing cones $C^{a}$ and $C^{s}$. Then $L$ restricted to $P_{-1}^{a}$ is $u_{0}$-positive with $u_{0}(t)=\sin \pi t$; and $L$ restricted to $P_{-1}^{s}$ is $u_{0}$ - 
positive with $u_{0}(t)=\cos \pi t$.

Proof. Consider only $P_{-1}^{a}$. From reduction by symmetry, if $z \in P_{-1}^{a}$ then (6.3) can be written as

$$
(L z)(t)=\frac{1}{2 \varepsilon} \int_{|t-\varepsilon|}^{1-|1-t-\varepsilon|} g^{\prime}\left(x_{\varepsilon}(\tau)\right) z(\tau) d \tau
$$

provided $\tau \in(0,1)$. Recall that the kernel $g^{\prime}\left(x_{\varepsilon}(\tau)\right)$ in (6.4) is positive everywhere and that the interval of integration in (6.4) is contained in $[0,1]$. As in the proof of Proposition 3.4, if $z \in C^{a}$ is strictly positive on some interval $\left(a_{0}, b_{0}\right) \subseteq(0,1)$, then $\left(L^{n} z\right)(t)>0$ on an interval $\left(a_{n}, b_{n}\right)$ where $\left(a_{n}, b_{n}\right)=(0,1)$ for some $n$. For this value of $n$ we easily see that $L^{n+1} z$ is also positive throughout $(0,1)$ and in addition has simple zeros at the endpoints $t=0,1$. Such a function can then be bounded above and below by suitable positive multiples of $\sin \pi t$.

PROPOSITION 6.3. The restriction of $L$ to $P_{-1}^{s}$ has $\alpha=1$ as an algebraically simple eigenvalue with corresponding eigenvector $x_{\varepsilon}^{\prime}$, and $|\alpha|<1$ for all other eigenvalues. The restriction of $L$ to $P_{-1}^{a}$ has spectral radius less than one.

COROLLARY 6.4. The only solution (up to scalar multiple) of the linear equation (LE) in $P_{-1}$ is $x_{\varepsilon}^{\prime}(t)$.

We prove only Proposition 6.3 as the Corollary follows easily.

Proof of Proposition 6.3. The claim about $L$ restricted to $P_{-1}^{s}$ follows immediately from Theorem 6.1 as $L x_{\varepsilon}^{\prime}=x_{\varepsilon}^{\prime}$ belongs to the cone $C^{s}$.

To prove that $L$ restricted to $P^{a}{ }_{-1}$ has spectral radius less than one, first differentiate $L x_{\varepsilon}^{\prime}=x_{\varepsilon}^{\prime}$ in (6.3) to obtain

$$
x_{\varepsilon}^{\prime \prime}(t)=\frac{1}{2 \varepsilon} \int_{-\varepsilon}^{\varepsilon} g^{\prime}\left(x_{\varepsilon}(t-\tau)\right) x_{\varepsilon}^{\prime \prime}(t-\tau) d \tau-h(t)
$$

where

$$
h(t)=-\frac{1}{2 \varepsilon} \int_{-\varepsilon}^{\varepsilon} g^{\prime \prime}\left(x_{\varepsilon}(t-\tau)\right)\left[x_{\varepsilon}^{\prime}(t-\tau)\right]^{2} d \tau .
$$

This can be written abstractly as

$$
(I-L)\left(-x_{\varepsilon}^{\prime \prime}\right)=h
$$

where both $-x_{\varepsilon}^{\prime \prime}$ and $h$ belong to $C^{a}$.

Now let $\lambda_{0}$ denote the spectral radius of $L$ restricted to $P_{-1}^{a}$, and let $F_{0}^{a} \in C^{a *} \backslash\{0\}$ denote the unique eigenvector of the adjoint $L^{*}$ corresponding to the eigenvalue $\lambda_{0}$, as given by Theorem 6.1. Taking the duality pairing $\langle$,$\rangle of (6.5) with F_{0}^{a}$ now gives

$$
\left(1-\lambda_{0}\right) c_{1}=c_{2}
$$

where $c_{1}=\left\langle F_{0}^{a},-x_{\varepsilon}^{\prime \prime}\right\rangle$ and $c_{2}=\left\langle F_{0}^{a}, h\right\rangle$ are positive. It follows now that $\lambda_{0}<1$, as 
required.

We now eliminate solutions of the form (6.2) for $\mu=0, p=x_{\varepsilon}^{\prime}$.

Proposition 6.5. Equation (LE) does not have a solution of the form

$$
z(t)=t x_{\varepsilon}^{\prime}(t)+v(t), \quad \text { with } \quad v(t+2)=v(t) .
$$

Proof. Substituting (6.6) into (6.3) and making use of $x_{\varepsilon}^{\prime}=L x_{\varepsilon}^{\prime}$ gives $v=L v-k$ where

$$
k(t)=\frac{1}{2 \varepsilon} \int_{1-\varepsilon}^{1+\varepsilon} f^{\prime}\left(x_{\varepsilon}(t-\tau)\right) \tau x_{\varepsilon}^{\prime}(t-\tau) d \tau .
$$

Since $k \in P_{-1}$, without loss $v \in P_{-1}$ (otherwise replace $v$ with its projection onto $P_{-1}$ ). As the operator $L$ commutes with the projection onto $P^{s}$, we may further replace $v$ and $k$ with their projections $v^{s}$ and $k^{s}$ onto $P_{-1}^{s}$. Thus

$$
v^{s}=L v^{s}-k^{s}
$$

where a calculation reveals

$$
k^{s}(t)=\frac{1}{2}[k(t)+k(-t)]=x_{\varepsilon}^{\prime}(t) .
$$

Now the inhomogeneous equation (6.7) has a solution if and only if $\left\langle F_{0}^{s}, k^{s}\right\rangle=0$ where $F_{0}^{s}$ is the unique eigenvector of the adjoint $L^{*}$ corresponding to the eigenvalue $\alpha=1$ and the symmetry class $P_{-1}^{s}$, and as before $\langle$,$\rangle is the duality pairing. One$ easily verifies that

$$
\left(L^{*} z\right)(t)=\frac{1}{2 \varepsilon} f^{\prime}\left(x_{\varepsilon}(t)\right) \int_{1-\varepsilon}^{1+\varepsilon} z(t-\tau) d \tau
$$

and

$$
F_{\theta}^{s}(t)=f^{\prime}\left(x_{\varepsilon}(t)\right) x_{\varepsilon}^{\prime}(t) \text {. }
$$

(The transformation $L^{*}$ is actually defined on the dual space whose elements are measures. In the above two formulas we make a slight abuse of notation by interpreting integrable functions as measures.) It follows that

$$
\left\langle F_{0}^{s}, k^{s}\right\rangle=\int_{0}^{1} F_{0}^{s}(t) x_{\varepsilon}^{\prime}(t) d t=\int_{0}^{1} f^{\prime}\left(x_{\varepsilon}(t)\right)\left[x_{\varepsilon}^{\prime}(t)\right]^{2} d t<0
$$

so (6.7) has no solution.

Proposition 6.5 implies that if $\alpha=1$ is not an algebraically simple characteristic multiplier, then equation (LE) must have a two-periodic solution $z$ independent of $x_{\varepsilon}^{\prime}$. If this is so, then from Corollary 6.4 and the identity $f^{\prime}\left(x_{\varepsilon}(t+1)\right)=f^{\prime}\left(x_{\varepsilon}(t)\right)$, we may assume without loss that such a solution $z$ lies in the symmetry class $P_{1}$; for if not, we may consider the solution $z(t)+z(t+1)$ instead. In Lemma 9.3 we shall eliminate the 
possibility that $z \in P_{1}$, at least for $\varepsilon \leqslant 1 / 3$, and thereby conclude that the characteristic multiplier $\alpha=1$ is algebraically simple.

\section{Dependence of $x_{\varepsilon}$ on $\varepsilon$}

The operator $I-L$ is invertible on the space $P_{-1}^{a}$ by Proposition 6.3. As this is the Fréchet derivative of the nonlinear operator $x \rightarrow x-T_{\varepsilon} x$ with respect to $x \in P_{-1}^{a}$, it should follow from the Implicit Function Theorem that the solution $x_{\varepsilon}$ obtained by monotone iteration depends smoothly on $\varepsilon$. Certainly this is the case if $T$ depends smoothly on $x$ and $\varepsilon$.

We shall show $T$ is a $C^{1}$ function of $(x, \varepsilon) \in P^{a}{ }_{-1} \times(0, \infty)$. Actually, if $\varepsilon$ is fixed then $T_{\varepsilon}$ is a $C^{k}$ function of $x \in P^{a}{ }_{-1}$, provided $f$ is $C^{k}$. But a subtle point is that $T$ is never jointly $C^{2}$ in $(x, \varepsilon)$ no matter how smooth $f$ is. Thus the Implicit Function Theorem by itself yields only the result that $x_{\varepsilon}$ is a $C^{1}$ function of $\varepsilon$. Following Hale [10], we shall show, however, that repeated use of the Implicit Function Theorem proves that in fact $x_{\varepsilon}$ is a $C^{k}$ function of $\varepsilon$ if $f$ is $C^{k}$.

The reason for the difficulty about smoothness is due to the fact that varying $\varepsilon$ is, in some sense, like applying a translation operator. To be specific consider $S_{\varepsilon}$ : $P_{-1}^{a} \rightarrow P_{-1}^{a}$ given by

$$
\left(S_{\varepsilon} x\right)(t)=\int_{-\varepsilon}^{\varepsilon} g(x(t-\tau)) d \tau .
$$

Note that $S_{\varepsilon}=2 \varepsilon T_{\varepsilon}$. At least formally, the second derivative of $S_{\varepsilon} x$ with respect to $\varepsilon$ is the function

$$
\left[\frac{d^{2} S_{\varepsilon} x}{d \varepsilon^{2}}\right](t)=\frac{d}{d \varepsilon}[g(x(t-\varepsilon))+g(x(t+\varepsilon))]
$$

however, this function does not exist for those $x \in P^{a}{ }_{-1}$ which are not $C^{1}$. Moreover, casting the problem in a space of smoother functions is no remedy. The following result is essentially the sharpest possible on the smoothness of the map $S$ jointly in $(x, \varepsilon)$.

LEMMA 7.1. The operator $S$ in (7.1) defines a $C^{1}$ mapping from $P_{-1}^{a} \times(0, \infty)$ into $P_{-1}^{a}$.

Proof. The candidate for Fréchet derivative $D S_{\varepsilon} x$ of $S_{\varepsilon}$ at some $(x, \varepsilon) \in P_{-1}^{a} \times$ $(0, \infty)$ is the linear operator $\Phi(x, \varepsilon)$ from $P_{-1}^{a} \times \boldsymbol{R}$ into $P_{-1}^{a}$, defined by

$$
\Phi(x, \varepsilon)(y, \delta)=\int_{-\varepsilon}^{\varepsilon} g^{\prime}(x(t-\tau)) y(t-\tau) d \tau+\delta[g(x(t-\varepsilon))+g(x(t+\varepsilon))] .
$$

That this is the derivative can be seen by estimating the difference 


$$
\begin{aligned}
S_{\varepsilon+\delta}(x+y)-S_{\varepsilon} x-\Phi(x, \varepsilon)(y, \delta)= & \left.\int_{-\varepsilon-\delta}^{\varepsilon+\delta}\left[g(x+y)-g(x)-g^{\prime}(x) y\right]\right|_{t-\tau} d \tau \\
& +\int_{\varepsilon}^{\varepsilon+\delta} g(x(t-\tau))-g(x(t-\varepsilon))+g^{\prime}(x(t-\tau)) y(t-\tau) d \tau \\
& +\int_{-\varepsilon-\delta}^{-\varepsilon} g(x(t-\tau))-g(x(t+\varepsilon))+g^{\prime}(x(t-\tau)) y(t-\tau) d \tau
\end{aligned}
$$

for a fixed $(x, \varepsilon)$ : using the uniform continuity of $x$, one shows the norm of this difference is order $o(\|y\|+|\delta|)$ as $(y, \delta) \rightarrow(0,0)$. Hence $D S_{\varepsilon} x=\Phi(x, \varepsilon)$ at each $(x, \varepsilon)$.

To show that $S$ is $C^{1}$, jointly in $(x, \varepsilon)$, we must show that $\Phi(x, \varepsilon)$ varies continuously as a linear operator, as $(x, \varepsilon)$ varies. This is a consequence of the following estimate of the operator norm:

$$
\begin{aligned}
\left\|\Phi\left(x_{2}, \varepsilon_{2}\right)-\Phi\left(x_{1}, \varepsilon_{1}\right)\right\| \leqslant & \sup _{t} \int_{-\varepsilon_{2}}^{\varepsilon_{2}}\left|g^{\prime}\left(x_{2}(t-\tau)\right)-g^{\prime}\left(x_{1}(t-\tau)\right)\right| d \tau \\
& +\sup _{t}\left(\int_{\varepsilon_{1}}^{\varepsilon_{2}}+\int_{-\varepsilon_{2}}^{-\varepsilon_{1}}\right)\left|g^{\prime}\left(x_{1}(t-\tau)\right)\right| d \tau \\
& +\sup _{t}\left|g\left(x_{2}\left(t-\varepsilon_{2}\right)\right)-g\left(x_{1}\left(t-\varepsilon_{1}\right)\right)\right| \\
& +\sup _{t}\left|g\left(x_{2}\left(t+\varepsilon_{2}\right)\right)-g\left(x_{1}\left(t+\varepsilon_{1}\right)\right)\right| .
\end{aligned}
$$

As the above tends to zero when $\left(x_{2}, \varepsilon_{2}\right) \rightarrow\left(x_{1}, \varepsilon_{1}\right)$, the result is proved.

Lemma 7.1 and the discussion above imply that $\varepsilon \longmapsto x_{\varepsilon}$ is $C^{1}$. Although $S$ is never smoother than $C^{1}$, the map $\varepsilon \rightarrow x_{\varepsilon}$ is in fact $C^{k}$ if $f$ is $C^{k}$. To prove this fact first differentiate the identity $S_{\varepsilon} x_{\varepsilon}-2 \varepsilon x_{\varepsilon}=0$ with respect to $\varepsilon$ to obtain $\Psi\left(\partial x_{\varepsilon} / \partial \varepsilon, \varepsilon\right)=0$ where by definition

$$
\Psi(y, \varepsilon)=\Phi\left(x_{\varepsilon}, \varepsilon\right)(y, 1)-2 \varepsilon y-2 x_{\varepsilon},
$$

for $y \in P_{-1}^{a}$. We claim that $\Psi$ is $C^{1}$ if $g$ is $C^{2}$. Since $\Psi$ is linear in $y$ we concentrate on the differentiability with respect to $\varepsilon$. From the identity $S_{\varepsilon} x_{\varepsilon}-2 \varepsilon x_{\varepsilon}=0$ it follows easily that $\left(\partial x_{\varepsilon} / \partial t\right)(t)$ exists and depends continuously on $\varepsilon$, uniformly in $t$. Hence $\varepsilon \longmapsto x_{\varepsilon}(\cdot-\varepsilon)$ and $\varepsilon \longmapsto x_{\varepsilon}(\cdot+\varepsilon)$ are $C^{1}$ mappings taking values in $P_{2}$ and our claim follows at once. Next, applying the implicit function theorem to $\Psi$, we find that the solution $y=\partial x_{\varepsilon} / \partial \varepsilon$ is $C^{1}$ in $\varepsilon$ if $g$ is $C^{2}$. Repeating the argument (note that $\Psi\left(\partial x_{\varepsilon} / \partial \varepsilon, \varepsilon\right)=0$ implies that $\partial^{2} x_{\varepsilon} / \partial t \partial \varepsilon$ exists and depends continuously on $\varepsilon$ ) we obtain the following result.

THEOREM 7.2. If $f$ is $C^{k}$ then the two-periodic solution $x_{\varepsilon}$ is a $C^{k}$ function of $\varepsilon<\varepsilon^{*}$. So are the solutions $x_{k \varepsilon}(k t)$ obtained in Theorems $3.8,3.10$ and 3.12 for the appropriate ranges of $\varepsilon$. 


\section{Slow Oscillations Yield Dominant Multipliers}

In this section we consider the linear equation

$$
z(t)=\int_{1-\varepsilon}^{1+\varepsilon} r(t-\tau) z(t-\tau) d \tau
$$

where we assume that the continuous function $r$ satisfies

$$
r(t+2)=r(t)<0
$$

for all $t$. For example $r(t)=(1 / 2 \varepsilon) f^{\prime}\left(x_{\varepsilon}(t)\right)$ would give the variational equation (LE). We shall single out a class of solutions defined for all $t \in(-\infty, \infty)$, the "slowly oscillating solutions", characterized by the property that all zeros are simple and are spaced at least a distance $1-\varepsilon$ apart. For example the solution $z(t)=x_{\varepsilon}^{\prime}(t)$ of (LE) is slowly oscillating, while the rescaled solution $z(t)=x_{k \varepsilon}^{\prime}(k t)$ for $k=3,5,7, \cdots$ are not if the spacing $1 / k$ between zeros is less than $1-\varepsilon$ (which is certainly the case if $\varepsilon<\varepsilon^{*} / k<1 / 3$ ).

As noted in the Introduction, a restriction in our methods is the requirement that $\varepsilon \leqslant 1 / 3$. This arises from the fact that in many of the proofs the mimimum separation $1-\varepsilon$ of zeros of slowly oscillating solutions must not be less than the length $2 \varepsilon$ of the interval of integration in (8.1). That is, we require that $1-\varepsilon \geqslant 2 \varepsilon$. How essential this restriction is for our final results is an open question.

Observe that because $r(t)<0$, no solution of $(8.1)$ can be non-zero on an open interval of length $1+\varepsilon$; indeed all solutions must "oscillate", that is, change sign infinitely often. We shall show that at most two Floquet multipliers (counting multiplicity) can have slowly oscillating eigenfunctions and that these multipliers must be dominant, i.e., have norms strictly larger than all others. From this, stability results for solutions of the nonlinear equation (E) will be obtained.

Let $z(t)$ be a solution of $(8.1)$ for all $t \in(-\infty, \infty)$. We say $z$ is slowly oscillating at $t_{0}$ in case all zeros of $z$ in the closed interval $\left[t_{0}-1-\varepsilon, t_{0}\right]$ are simple (i.e. $z(t)=0$ implies $\left.z^{\prime}(t) \neq 0\right)$ and are spaced a distance $1-\varepsilon$ or more apart. As noted, $r(t)<0$ forces $z$ to have at least one zero in this interval. If $\varepsilon<1 / 3$ then $2(1-\varepsilon)>1+\varepsilon$, so there can be at most two zeros. This conclusion is also true if $\varepsilon=1 / 3$, but is a little harder to prove. Clearly $\left[t_{0}-1-\varepsilon, t_{0}\right]=\left[t_{0}-4 / 3, t_{0}\right]$ has at most three zeros of $z$; if exactly three, they must be located at $t_{0}, t_{0}-2 / 3$, and $t_{0}-4 / 3$, spaced a distance $1-\varepsilon=2 / 3$ apart. But then (8.1) would force $z\left(t_{0}\right) \neq 0$, a contradiction. To summarize: if $z$ is slowly oscillating at $t_{0}$, and if $\varepsilon \leqslant 1 / 3$, then the interval $\left[t_{0}-1-\varepsilon, t_{0}\right]$ contains either one or two zeros of $z$.

We say $z$ is slowly oscillating if it is slowly oscillating at each $t_{0} \in(-\infty, \infty)$. We say $z$ is never slowly oscillating if it is slowly oscillating at no $t_{0} \in(-\infty, \infty)$.

From now on we assume $\varepsilon \leqslant 1 / 3$. With this condition we can regard consecutive zeros $t_{n-1}<t_{n}$ of a slowly oscillating solution as being in a mother-daughter relationship: as $z\left(t_{n}\right)=0$, by (8.1) $z$ must vanish at some $t_{*} \in\left(t_{n}-1-\varepsilon, t_{n}-1+\varepsilon\right)$; and $t_{*}=t_{n-1}$ (the "mother" of $t_{n}$ ) is unique because the length $2 \varepsilon$ of this interval is no 
larger than the minimum separation $1-\varepsilon$ of zeros. And further, $t_{n}$ "gives birth" to the next zero $t_{n+1} \in\left(t_{n}+1-\varepsilon, t_{n}+1+\varepsilon\right)$ as the integrand in (8.1) changes sign as $t$ ranges in this interval. These ideas in fact imply the following important property.

PROPOSITION 8.1. If $z$ is slowly oscillating at $t_{0}$, then it is slowly oscillating at each $t_{1}>t_{0}$.

Proof. If not, let $t_{1}>t_{0}$ be the first point at which $z$ is not slowly oscillating. (Clearly $z$ is slowly oscillating immediately to the right of $t_{0}$, so $t_{1}$ does exist.) It is also clear from the definitions that $z\left(t_{1}\right)=0$; hence from (8.1) $z$ must vanish at some $t_{*} \in\left(t_{1}-1-\varepsilon, t_{1}-1+\varepsilon\right)$. Because $z$ is slowly oscillating immediately to the left of $t_{1}$, and because $2 \varepsilon \leqslant 1-\varepsilon$, it follows that besides $t_{*}$ there exists at most one other zero $t_{* *}$ of $z$ in the interval $\left[t_{1}-1-\varepsilon, t_{1}\right.$ ), that $t_{*}$ and $t_{* *}$ are simple zeros, and that $t_{* *}$ (if it exists) does not lie in $\left[t_{1}-1-\varepsilon, t_{1}-1+\varepsilon\right]$. In particular, $z$ has opposite signs at $t_{1}-1-\varepsilon$ and $t_{1}-1+\varepsilon$, say

$$
\begin{aligned}
& z\left(t_{1}-1-\varepsilon\right)<0, \\
& z\left(t_{1}-1+\varepsilon\right)>0 .
\end{aligned}
$$

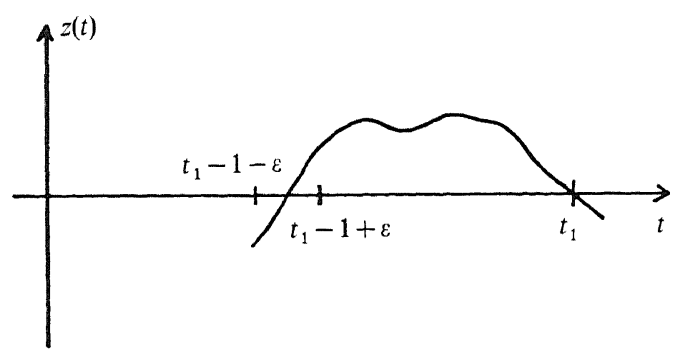

Fig. 9

Differentiating (8.1) we find

$$
z^{\prime}(t)=r(t-1+\varepsilon) z(t-1+\varepsilon)-r(t-1-\varepsilon) z(t-1-\varepsilon)
$$

so we see from this that $z^{\prime}\left(t_{1}\right)<0$. Hence $z$ is positive immediately to the left of $t_{1}$; because $z\left(t_{1}-1+\varepsilon\right)$ also is positive, it follows that $t_{* *}$ does not exist. See Fig. 9. Thus $t_{*}$ and $t_{1}$ are the only zeros of $z$ in $\left[t_{1}-1-\varepsilon, t_{1}\right]$ and both are simple. Hence $z$ is slowly oscillating at $t_{1}$, a contradiction.

The characteristic multipliers $\alpha \neq 0$ of equation (8.1) are the eigenvalues of the linear operator $\Lambda$ on $C[-1-\varepsilon, 0]$ which takes an initial condition $z(\tau)$, defined for $-1-\varepsilon \leqslant \tau \leqslant 0$, to its time two translate $z(\tau+2)$. Consider the corresponding eigenspace and generalized eigenspace; they correspond to the following spaces $E_{\alpha} \subseteq G_{\alpha}$ of complex solutions on $(-\infty, \infty)$ :

$$
\begin{aligned}
& E_{\alpha}=\{z: R \rightarrow C \mid z(t) \text { is a solution of }(8.1) \text { of the form } \\
& \left.\qquad z(t)=e^{\mu t} p(t) \text {, where } \alpha=e^{2 \mu} \text { and } p(t+2) \equiv p(t)\right\},
\end{aligned}
$$




$$
\begin{aligned}
& G_{a}=\{z: \mathbb{R} \rightarrow C \mid z(t) \text { is a solution of }(8.1) \text { of the form } \\
&\left.z(t)=e^{\mu t} \sum_{j=0}^{k} t^{j} p_{j}(t) \text { for some } k, \text { where } \alpha=e^{2 \mu} \text { and } p_{j}(t+2) \equiv p_{j}(t) \text { for each } j\right\} .
\end{aligned}
$$

Floquet theory implies that the function $e^{\mu t} p_{k}(t)$ obtained from the leading term of any $z \in G_{\alpha}$ always belongs to $E_{\alpha}$.

If $\sigma>0$, define also the spaces of real solutions

$$
\begin{aligned}
& \mathscr{E}_{\sigma}=\operatorname{Re} \operatorname{span}_{|\alpha|=\sigma} E_{\alpha}, \\
& \mathscr{G}_{\sigma}=\operatorname{Re} \operatorname{span}_{|\alpha|=\sigma} G_{\alpha}, \\
& \mathscr{H}_{\sigma}=\operatorname{Re} \operatorname{span}_{|\alpha| \geqslant \sigma} G_{\alpha},
\end{aligned}
$$

consisting of the real parts of the linear spans of various eigenspaces. All of these spaces are finite dimensional. Observe also that $\mathscr{E}_{\sigma} \subseteq \mathscr{G}_{\sigma} \subseteq \mathscr{H}_{\sigma}$ and that $\mathscr{H}_{\sigma_{1}} \subseteq \mathscr{H}_{\sigma_{2}}$ if $\sigma_{1}>\sigma_{2}$. Furthermore, each $z \in \mathscr{E}_{\sigma}$ has the form

$$
z(t)=e^{\rho t} q(t)
$$

and each $z \in \mathscr{G}_{\sigma}$ the form

$$
z(t)=e^{\rho t} \sum_{j=0}^{k} t^{j} q_{j}(t)
$$

where $q$ and $q_{j}$ are quasi-periodic functions and $\sigma=e^{2 \rho}$. (Recall that a quasi-periodic function is one of the form

$$
q(t)=Q\left(\alpha_{1} t, \cdots, \alpha_{p} t\right)
$$

where $Q\left(\tau_{1}, \cdots, \tau_{p}\right)$ is continuous and of period one in each $\tau_{j}$, and the $\alpha_{j}$ are real constants. Without loss the $\alpha_{j}$ are linearly independent over the rationals.)

The relation between slowly oscillating solutions and the above eigenspaces is made clear by the following result.

PROPOSITION 8.2.

(i) Given $\sigma>0$, either each $z \in \mathscr{E}_{\sigma} \backslash\{0\}$ is slowly oscillating, or each such $z$ is never slowly oscillating.

(ii) If each $z \in \mathscr{E}_{\sigma} \backslash\{0\}$ is slowly oscillating, and $\mathscr{E}_{\sigma} \neq\{0\}$ (i.e. $\sigma=|\alpha|$ for some characteristic multiplier $\alpha$ ) then each $z \in \mathscr{H}_{\sigma} \backslash\{0\}$ is slowly oscillating.

(iii) If each $z \in \mathscr{H}_{\sigma} \backslash\{0\}$ is slowly oscillating then $\operatorname{dim} \mathscr{H}_{\sigma} \leqslant 2$; that is, there are at most two multipliers (counting multiplicity) with $|\alpha| \geqslant \sigma$.

Several lemmas are needed before the proof of Proposition 8.2 can be given. To begin, we show the property of being slowly oscillating is preserved under limits.

LEMMA 8.3. Let $\varepsilon_{n} \leqslant 1 / 3$ converge to $\varepsilon>0$, let $r_{n}(t)$ be a sequence of two-periodic 
functions converging uniformly to $r(t)<0$, and $z_{n}(t)$ a sequence of slowly oscillating solutions of

$$
z_{n}(t)=\int_{1-\varepsilon_{n}}^{1+\varepsilon_{n}} r_{n}(t-\tau) z_{n}(t-\tau) d \tau
$$

converging uniformly on compact intervals to $z(t)$. Then either $z$ is a slowly oscillating solution of the limiting equation (8.1), or $z$ is identically zero.

Proof. Each $z_{n}$ has only simple zeros, with consecutive zeros spaced a distance at least $1-\varepsilon_{n}$ and at most $1+\varepsilon_{n}$ apart; moreover the zeros of each $z_{n}$ form a bi-infinite sequence tending to $-\infty$ and $\infty$. By taking limits we easily conclude there are zeros $\left\{t_{k}\right\}_{k=-\infty}^{\infty}$ of $z$ so that for each $k$

$$
\left\{\begin{array}{l}
z\left(t_{k}\right)=0, \\
1-\varepsilon \leqslant t_{k+1}-t_{k} \leqslant 1+\varepsilon, \quad \text { and } \\
(-1)^{k} z(t) \geqslant 0 \quad \text { if } t \in\left(t_{k}, t_{k+1}\right) .
\end{array}\right.
$$

We must show either $z$ is identically zero, or else the zeros $t_{k}$ all are simple and there are no others.

Case I. $z$ vanishes identically on some interval $[a, b]$ of length $b-a \geqslant 2 \varepsilon$. Then, because $2 \varepsilon \leqslant 1-\varepsilon$, there exists $\tau \in[a, b]$ such that $I=(\tau-1-\varepsilon, \tau-1+\varepsilon)$ contains no $t_{k}$. Because $z$ is sign definite on $I$ (by (8.5)) we conclude from the integral equation (8.1) and $z(\tau)=0$ that

$$
z(t)=0 \quad \text { for each } \quad t \in[\tau-1-\varepsilon, \tau-1+\varepsilon] .
$$

Also, the vanishing of $z^{\prime}$ on $[a, b]$ implies, by (8.2), that

$$
r(t-1-\varepsilon) z(t-1-\varepsilon)=r(t-1+\varepsilon) z(t-1+\varepsilon) \quad \text { for each } \quad t \in[a, b] .
$$

From (8.6) and (8.7), and the fact that $\tau \in[a, b]$ it follows that $z$ vanishes identically on $[a-1-\varepsilon, b-1+\varepsilon]$, an interval of length $b-a+2 \varepsilon$.

The above argument may be repeated: $z$ vanishes identically on the interval $[a-1-\varepsilon, b-1+\varepsilon]$, hence also on the interval $[a-2-2 \varepsilon, b-2+2 \varepsilon]$. We conclude that for each positive integer $N, z$ vanishes identically on $[a-N(1+\varepsilon), b-N(1-\varepsilon)]$. Thus $z(t)$ vanishes for all small $t$, say for all $t \leqslant T$; but this implies $z$ is identically zero on $(-\infty, \infty)$ by uniqueness of solutions of $(8.1)$.

Case II. $z$ does not vanish identically on any interval of length $2 \varepsilon$ or more. If so, we may conclude

$$
z(t)=0 \text { implies } t \in\left(t_{k}+1-\varepsilon, t_{k}+1+\varepsilon\right) \text { for some } k .
$$

To see why (8.8) holds, suppose for some $t$ that $z(t)=0$, but that $(t-1-\varepsilon, t-1+\varepsilon)$ contains no $t_{k}$. The vanishing of the integral (8.1) and the sign condition (8.5) would then force $z$ to vanish identically on $[t-1-\varepsilon, t-1+\varepsilon]$; this is an interval of length $2 \varepsilon$, a contradiction. Thus (8.8) holds. 
Observe further that $z$ is monotone (not necessarily strictly) in each interval $I_{k}=$ $\left(t_{k}+1-\varepsilon, t_{k}+1+\varepsilon\right)$, as the derivative formula (8.2) and sign condition (8.5) imply that $(-1)^{k+1} z^{\prime}(t) \geqslant 0$ there. Also, these intervals are disjoint as $\varepsilon \leqslant 1 / 3$. Consequently for each $k$ the set of points $J_{k} \subseteq I_{k}$ at which $z$ vanishes is closed and connected.

Suppose now that $z$ is not slowly oscillating. If all zeros of $z$ are simple, then there must exist two of them spaced a distance less than $1-\varepsilon$ apart; but this is impossible in view of (8.5). Thus $z$ must possess a non-simple zero, say $z\left(t_{*}\right)=$ $z^{\prime}\left(t_{*}\right)=0$. From (8.8) we see that $I_{*}=\left(t_{*}-1-\varepsilon, t_{*}-1+\varepsilon\right)$ contains some $t_{k}$; moreover, this $k$ is unique. The sign of $z$ on either side of $t_{k}$ (given in (8.5)) and the derivative formula (8.2) at $t=t_{*}$ imply that $z\left(t_{*}-1-\varepsilon\right)=z\left(t_{*}-1+\varepsilon\right)=0$. And the vanishing of (8.1) at $t=t_{*}$ implies that $z$ must assume both positive and negative values in $I_{*}$ (note that $z$ does not vanish identically on this interval of length $2 \varepsilon$ ). Hence there exists $t_{* *} \in I_{*}$ such that $z\left(t_{* *}\right)=0$ and such that the three zeros $t_{*}-1-\varepsilon$, $t_{* *}, t_{*}-1+\varepsilon$ of $z$ all lie in different intervals $J_{j}$. But then $I_{*}$ intersects three different $I_{j}$ (since $J_{j} \subseteq I_{j}$ ); this is impossible as the intervals $I_{*}$ and $I_{j}$ all have the same length $2 \varepsilon$, and the intervals $I_{j}$ are pairwise disjoint.

LEMMA 8.4. If $z$ is slowly oscillating at $t_{0}$, and $z\left(t_{0}\right)=0$, then the zero $t_{1}<t_{0}$ immediately to the left of $t_{0}$ is at a distance strictly greater than $1-\varepsilon$. Thus, if $z$ is slowly oscillating then all its zeros are spaced a distance strictly greater than $1-\varepsilon$ apart.

Proof. If the first statement is false, then $t_{1}$ and $t_{0}$ are separated by a distance exactly $1-\varepsilon$, so that $z\left(t_{0}\right)=z\left(t_{0}-1+\varepsilon\right)=0$. But then $z(t) \neq 0$ throughout $\left(t_{0}-1-\varepsilon\right.$, $\left.t_{0}-1+\varepsilon\right)$ as the zero before $t_{1}=t_{0}-1+\varepsilon$ is a distance at least $1-\varepsilon \geqslant 2 \varepsilon$ away. Equation (8.1) then forces $z\left(t_{0}\right) \neq 0$, a contradiction. The second statement of the Lemma follows immediately from the first statement.

LEMMA 8.5. If $z \in \mathscr{E}_{\sigma} \backslash\{0\}$ then either $z$ is slowly oscillating, or is never slowly oscillating.

Proof. Suppose $z$ is slowly oscillating at some $t_{0}$; without loss $z\left(t_{0}\right)=0$ (if not, increase $t_{0}$ to the next zero). By Lemma 8.4 and because $2(1-\varepsilon) \geqslant 1+\varepsilon$, there is only one other zero of $z$ in $\left[t_{0}-1-\varepsilon, t_{0}\right]$ and it is a distance strictly greater than $1-\varepsilon$ from $t_{0}$. Now write $z$ in the form (8.3) with $q$ quasi-periodic. There exists a sequence $t_{n} \rightarrow-\infty$ such that

$$
\begin{aligned}
q\left(t+t_{n}\right) & \longrightarrow q(t) \\
q^{\prime}\left(t+t_{n}\right) & \longrightarrow q^{\prime}(t)
\end{aligned}
$$

uniformly on compact intervals; the convergence of $q$ follows from quasi-periodicity, and the convergence of $q^{\prime}$ because $q$ satisfies an integral equation. Therefore, using (8.3) we see that $e^{-\rho t_{n}} z\left(t+t_{n}\right)$ and $e^{-\rho t_{n}} z^{\prime}\left(t+t_{n}\right)$ converge to $z(t)$ and $z^{\prime}(t)$ uniformly on $\left[t_{0}-1-\varepsilon, t_{0}\right]$. It follows from the location and simplicity of the zeros of $z$ in this interval, that for large $n$ the solution $z$ is slowly oscillating at $t_{0}+t_{n} \rightarrow-\infty$. By Proposition $8.1, z$ is thus slowly oscillating. 
Proof of Proposition 8.2. Regard the finite dimensional space $\mathscr{E}_{\sigma}$ as isomorphic to $\boldsymbol{R}^{d}$. We show the set of slowly oscillating solutions $z \in \mathscr{E}_{\sigma} \backslash\{0\} \equiv \boldsymbol{R}^{d} \backslash\{0\}$ is both open and closed; then part (i) of the Proposition follows from this and Lemma 8.5. Now by Lemma 8.3 the set of slowly oscillating $z$ certainly is closed in $\mathscr{E}_{\sigma} \backslash\{0\}$. To see that it is open, let $z \in \mathscr{E}_{\sigma} \backslash\{0\}$ be slowly oscillating, and fix $t_{0}$ so that $z\left(t_{0}\right)=0$. Then as $z$ is slowly oscillating at $t_{0}$, and $2(1-\varepsilon) \geqslant 1+\varepsilon$, there can be only one other zero in $\left[t_{0}-1-\varepsilon, t_{0}\right]$, both it and $t_{0}$ are simple, and by Lemma 8.4 they must be a distance greater than $1-\varepsilon$ apart. From this it follows that nearby points in $\mathscr{E}_{\sigma}$ also are slowly oscillating at $t_{0}$; so by Lemma 8.5 they are slowly oscillating. This proves (i).

To show (ii), suppose each $z \in \mathscr{E}_{\sigma} \backslash\{0\}$ is slowly oscillating. We first show each $z \in \mathscr{G}_{\sigma} \backslash\{0\}$ is slowly oscillating, then show the same for each $z \in \mathscr{H}_{\sigma} \backslash\{0\}$. Each $z \in \mathscr{G}_{\sigma} \backslash\{0\}$ has the form (8.4) where the function $e^{\rho t} q_{k}(t)$ is slowly oscillating, as it is in $\mathscr{E}_{\sigma} \backslash\{0\}$. Writing $z(t)=e^{\rho t} t^{k}\left[q_{k}(t)+h(t)\right]$ where $h(t) \rightarrow 0$ as $t \rightarrow-\infty$, choosing $t_{0}$ so that $q_{k}\left(t_{0}\right)=0$, and $t_{n} \rightarrow-\infty$ so that $q_{k}\left(t+t_{n}\right) \rightarrow q_{k}(t)$, and noting $e^{-\rho t_{n}} t_{n}^{-k} z\left(t+t_{n}\right) \rightarrow e^{\rho t} q_{k}(t)$, we may argue as in the proof of Lemma 8.5 to show that $z$ is slowly oscillating at $t_{0}+t_{n}$ (for large $n$ ) because $e^{\rho t} q_{k}(t)$ is at $t_{0}$. As before, it follows that $z$ is slowly oscillating.

Now suppose each element of $\mathscr{G}_{\sigma} \backslash\{0\}$ is slowly oscillating; if we show each $z$ in a dense subset $\mathscr{D} \subseteq \mathscr{H}_{\sigma}$ is slowly oscillating, the same will hold for each $z \in \mathscr{H}_{\sigma} \backslash\{0\}$ by Lemma 8.3, and this will prove (ii). Let $\mathscr{D}$ consist of those $z=z_{0}+z_{1}$ where $z_{0} \in \mathscr{G}_{\sigma} \backslash\{0\}$ and $z_{1} \in \mathscr{H}_{\sigma_{1}}$ for some $\sigma_{1}>\sigma$. Clearly $\mathscr{D}$ is dense in $\mathscr{H}_{\sigma}$. Writing $z_{0}$ in the form (8.4), and noting $z_{1}$ decays faster at $-\infty$ than does $z_{0}$, that is, $z_{1}(t)=e^{\rho t} t^{k} h_{1}(t)$ with $h_{1}(t) \rightarrow 0$ as $t \rightarrow-\infty$, shows that $z(t)=e^{\rho t} t^{k}\left[q_{k}(t)+h(t)\right]$ where $h(t) \rightarrow 0$ as $t \rightarrow-\infty$. The rest of the proof follows the arguments of the paragraph above, using the fact that $e^{\rho t} q_{k}(t)$ belongs to $\mathscr{E}_{\sigma} \backslash\{0\}$ hence is slowly oscillating.

Lastly we prove (iii). If $\operatorname{dim} \mathscr{H}_{\sigma} \geqslant 3$ then an appropriate linear combination of three independent elements in this space yields some $z \in \mathscr{H}_{\sigma} \backslash\{0\}$ with $z(0)=z^{\prime}(0)=0$. But then $z$ would not be slowly oscillating at $t=0$.

We may now consider the maximal $\mathscr{H}_{\sigma}$ for which all $z \in \mathscr{H}_{\sigma} \backslash\{0\}$ are slowly oscillating, namely

$$
\mathscr{H}_{*}=\operatorname{span}\left\{\mathscr{H}_{\sigma} \mid \text { each } z \in \mathscr{H}_{\sigma} \backslash\{0\} \text { is slowly oscillating }\right\} .
$$

Three possibilities present themselves. Let $\left\{\alpha_{n}\right\}$ denote the characteristic multipliers of (8.1) counted with multiplicity, and ordered so that

$$
\left|\alpha_{1}\right| \geqslant\left|\alpha_{2}\right| \geqslant\left|\alpha_{3}\right| \geqslant \cdots \rightarrow 0 \text {. }
$$

(If there are only finitely many multipliers we consider $\alpha_{n}=0$ for large $n$.) Then either

(i) $\operatorname{dim} \mathscr{H}_{*}=2$, so $\operatorname{dim} \mathscr{H}_{*}=\mathscr{H}_{\left|\alpha_{2}\right|}$ and we have the strict inequality $\left|\alpha_{2}\right|>\left|\alpha_{3}\right|$; or

(ii) $\operatorname{dim} \mathscr{H}_{*}=1$, so $\mathscr{H}_{*}=\mathscr{H}_{\left|\alpha_{1}\right|}$ and $\left|\alpha_{1}\right|>\left|\alpha_{2}\right|$; or

(iii) $\mathscr{H}_{*}=\{0\}$.

The multipliers associated with $\mathscr{H}_{*}$ are called the principal multipliers. In case (i), $\alpha_{1}$ 
and $\alpha_{2}$ are the principal multipliers; in case (ii) $\alpha_{1}$ is the only principal multiplier, while in case (iii) there are no principal multipliers. Below we show that no principal multiplier lies in $(-\infty, 0)$. Subsequently we prove that the principal multipliers vary continuously under perturbations of $\varepsilon$ and the kernel $r(t)$ in (8.1), and are preserved under limits; this means case (i) is preserved under perturbations, and under limits provided $\left|\alpha_{2}\right|$ does not tend to zero. We believe case (i) always holds.

PROPOSITION 8.6. No principal multiplier lies in $(-\infty, 0)$.

Proof. If this is false, then there exists a slowly oscillating solution satisfying $z(t+2)=-|\alpha| z(t)$, where $\alpha \in(-\infty, 0)$ is a principal multiplier. Let $z\left(t_{0}\right)=0$ and, say, $z^{\prime}\left(t_{0}\right)>0$. The interval $\left[t_{0}, t_{0}+2\right]$ must contain zeros other than $t_{0}+2$, as the maximum separation of zeros is $1+\varepsilon<2$. There must exist at least two others because $z^{\prime}\left(t_{0}\right)$ and $z^{\prime}\left(t_{0}+2\right)$ have opposite signs and all zeros of $z$ are simple. It follows that there exist two zeros of $z$ in $\left[t_{0}, t_{0}+2\right]$ a distance $2 / 3$ or less apart. But $2 / 3 \leqslant 1-\varepsilon$, so this contradicts Lemma 8.4.

PROPOSITION 8.7. Let $\operatorname{dim} \mathscr{H}_{*}=2$ hold for (8.1), namely that each $z \in \mathscr{H}_{\left|\alpha_{2}\right|} \mid\{0\}$ is slowly oscillating. Then there exists $\delta>0$ such that $\operatorname{dim} \mathscr{H}_{*}=2$ for any perturbed equation $z(t)=\int_{1-\varepsilon_{0}}^{1+\varepsilon_{0}} r_{0}(t-\tau) z(t-\tau) d \tau$ such that $\left|\varepsilon-\varepsilon_{0}\right| \leqslant \delta$ and $\left|r_{0}(t)-r(t)\right| \leqslant \delta$ for all $t$.

Proof. Standard results of spectral theory applied to the time two translation operator $\Lambda$ imply that the first two multipliers $\alpha_{01}, \alpha_{02}$ of the perturbed equation are near those of the unperturbed equation (8.1), and that the subspace $\mathscr{H}_{\left|\alpha_{02}\right|}$ for the perturbed equation has a basis $z_{01}(t), z_{02}(t)$ which is uniformly near a given basis $z_{1}(t)$, $z_{2}(t)$ of $\mathscr{H}_{\left|\alpha_{2}\right|}$ on the interval $\left[-1-\varepsilon_{0}, 0\right]$. Indeed, $z_{0 n}$ and its derivative $z_{0 n}^{\prime}$ are uniformly near $z_{n}$ and $z_{n}^{\prime}$ on compact intervals, for $n=1,2$, as these functions are exponential-polynomial-periodic functions which are solutions of an integral equation. It follows from Lemma 8.4 that $z_{0 n}$ is slowly oscillating because $z_{n}$ is. Hence $\operatorname{dim} \mathscr{H}_{*}=2$ holds for the perturbed equation.

PROPOSITION 8.8. Let $\operatorname{dim} \mathscr{H}_{*}=2$ hold for each equation

$$
z(t)=\int_{1-\varepsilon_{n}}^{1+\varepsilon_{n}} r_{n}(t-\tau) z(t-\tau) d \tau,
$$

where $r_{n}$ is two-periodic, $r_{n}(t) \rightarrow r(t)<0$ uniformly and $\varepsilon_{n} \leqslant 1 / 3$ converges to $\varepsilon>0$. If the corresponding multipliers $\alpha_{n 2}$ satisfy $\lim _{n \rightarrow \infty} \alpha_{n 2} \neq 0$ (this limit necessarily exists) then for the limiting equation (8.1) we also have $\operatorname{dim} \mathscr{H}_{*}=2$.

Proof. The corresponding time-two translation operators $\Lambda_{n}$ converge to $\Lambda$. So $\operatorname{dim} \mathscr{H}_{\left|\alpha_{2}\right|} \geqslant 2$ and, moreover, we can find at least one sequence of eigenvectors in $\mathscr{H}_{\left|\alpha_{n_{2} \mid}\right|}$ which converges to an eigenvector $z$ in $\mathscr{H}_{\left|\alpha_{2}\right|}$, uniformly on $[-1-\varepsilon, 0]$. Lemma 8.3 implies that $z$ is slowly oscillating. But then it follows from Proposition 8.2 that every element of $\mathscr{H}_{\left|\alpha_{2}\right|}$ is slowly oscillating and that, actually, dim $\mathscr{H}_{\left|\alpha_{2}\right|}=$ 2. 


\section{The Stability of $x_{\varepsilon}$}

The theory developed in Section 8 and the results of Section 6 will be used to prove the following results.

THEOREM 9.1. If $\varepsilon \leqslant 1 / 3$ then the solution $x_{\varepsilon}$ is asymptotically stable.

THEOREM 9.2. For $k \geqslant 3$ odd and any $\varepsilon<\varepsilon^{*} / k<1 / 3$, the solution $x_{k \varepsilon}(k t)$ of $(\mathrm{E})$ is unstable. The same is true if $\varepsilon_{n}^{-} / k<\varepsilon<\varepsilon_{n}^{+} / k, k+n$ is even, and $\varepsilon \leqslant 1 / 3$.

The proofs of these theorems follow from the results below.

Consider first the solution $x_{\varepsilon}$ for $0<\varepsilon<\varepsilon^{*}$; assume that $\varepsilon \leqslant 1 / 3$ so that the theory of Section 8 applies. Then $x_{\varepsilon}^{\prime}$ is a slowly oscillating solution of the linearized equation (LE); this is by (ii) of Theorem 3.6. Hence $\alpha=1$ is a principal multiplier. If there are no other principal multipliers (that is, $\operatorname{dim} \mathscr{H}_{*}=1$ ) then $|\alpha|<1$ for all other characteristic multipliers of (LE) and so $x_{\varepsilon}$ is asymptotically stable. On the other hand, if $\operatorname{dim} \mathscr{H}_{*}=2$, then there exists one other principal multiplier $\beta$. Necessarily, $\beta$ is real. Further, $\beta>0$ by Proposition 8.6. And Propositions 8.7 and 8.8 imply that $\beta=\beta(\varepsilon)$ depends continuously on $\varepsilon$, and continues to exist for as long as it remains bounded away from zero. From the following result we conclude $\beta \neq 1$, and so $\beta \in(0,1) \cup(1, \infty)$.

LEMMA 9.3. The characteristic multiplier $\alpha=1$ for $x_{\varepsilon}$ is algebraically simple when $\varepsilon \leqslant 1 / 3$.

Proof. The linear equation (LE) has the two-periodic solution $x_{\varepsilon}^{\prime}$. By Proposition 6.5 there is no solution of the form $t x_{\varepsilon}^{\prime}(t)+v(t)$ where $v$ is two-periodic. Therefore the only way the multiplier $\alpha=1$ could fail to be simple is if there was a two-periodic solution $z$ of (LE), independent from $x_{\varepsilon}^{\prime}$. By (i) of Proposition 8.2 we see that $z$ is slowly oscillating because $x_{\varepsilon}^{\prime}$ is, and because both $x_{\varepsilon}^{\prime}$ and $z$ belong to $\mathscr{E}_{1} \backslash\{0\}$. Also, by considering $z(t) \pm z(t+1)$, which are themselves solutions of (LE), we may assume without loss that either $z \in P_{1}$ or $z \in P_{-1}$. By Corollary $6.4, z \in P_{-1}$ is impossible, so necessarily $z \in P_{1}$. That is, $z$ must have period one. But $z \in P_{1}$ is impossible for a slowly oscillating solution: let $z\left(t_{0}\right)=0$, and so $z^{\prime}\left(t_{0}\right) \neq 0$. By periodicity $z$ must vanish at some point in $\left(t_{0}, t_{0}+1\right)$, hence it must have two zeros spaced a distance no more than $1 / 2$ apart; and $1 / 2<1-\varepsilon$, contradicting the definition of slowly oscillating.

Lemma 9.3 implies that one of two situations holds as $\varepsilon$ varies in the interval $\left(0, \varepsilon^{*}\right) \cap(0,1 / 3]$ :

(i) $\beta(\varepsilon)>1$ for all such $\varepsilon$, in which case $x_{\varepsilon}$ is unstable; or

(ii) for each such $\varepsilon$ either $0<\beta(\varepsilon)<1$ or else $\operatorname{dim} \mathscr{H}_{*}=1$; here $x_{\varepsilon}$ is asymptotically stable.

We will show that (ii) holds, thereby proving Theorem 9.1. Indeed, it is sufficient to show this only for a particular nonlinearity $f^{0}$ satisfying the hypothesis $H_{1}-H_{4}$. For if $f$ is any other such function, consider the homotopy $f^{\rho}=\rho f+(1-\rho) f^{0}$ for $0 \leqslant \rho \leqslant 1$. 
We obtain then a two-parameter family $x_{\varepsilon, \rho}$ of solutions, for $0<\varepsilon<\varepsilon^{*}(\rho)$ where $\varepsilon^{*}(\rho)>0$ is the bifurcation point for the integral equation for $f^{\rho}$. As $(\varepsilon, \rho)$ ranges over the connected set

$$
\Sigma=\left\{(\varepsilon, \rho) \mid 0<\varepsilon<\varepsilon^{*}(\rho), \varepsilon \leqslant 1 / 3,0 \leqslant \rho \leqslant 1\right\}
$$

the principal multiplier $\beta(\varepsilon, \rho)$ varies continuously in $[0,1) \cap(1, \infty)$. (We interpret $\beta=0$ as meaning $\operatorname{dim} \mathscr{H}_{*}=1$, i.e., $\alpha=1$ is the only principal multiplier.) Thus either $x_{\varepsilon, \rho}$ is unstable for all $(\varepsilon, \rho) \in \Sigma$ or it is asymptotically stable for all $(\varepsilon, \rho) \in \Sigma$.

Hence it is enough to show asymptotic stability for a particular example. Consider the cubic polynomial

$$
f^{0}(x)=-(1+\gamma) x+\gamma x^{3} \quad \text { for }|x| \leqslant 1,
$$

where the parameter $\gamma$ is fixed. It is easy to see that if $0<\gamma<1 / 2$ then $f^{0}$ can be extended to $(-\infty, \infty)$ in such a way that hypotheses $H_{1}$ through $H_{4}$ are all satisfied. Remarkably, for the parameter value $\varepsilon=1 / 3$ and for $\gamma$ in a certain range, an explicit expression for the solution $x_{\varepsilon}$ can be given. This explicit form of $x_{\varepsilon}$ will aid in the stability analysis.

PROPOSITION 9.4. If $\varepsilon=1 / 3$ and if $\gamma_{0}<\gamma<1 / 2$, where $\gamma_{0}=2 \pi / 3 \sqrt{3}-1 \simeq .2092$, for the nonlinearity (9.1), the solution $x_{\varepsilon}$ is exactly

$$
x_{\varepsilon}(t)=\left[\frac{4}{3}\left(1-\frac{\gamma_{0}}{\gamma}\right)\right]^{1 / 2} \sin \pi t .
$$

Proof. We first clarify the role of the constant $\gamma_{0}$ by observing that, as $\varepsilon^{*}$ satisfies $\sin \pi \varepsilon^{*} / \pi \varepsilon^{*}=1 /(1+\gamma)$, we have $1 / 3 \in\left(0, \varepsilon^{*}\right)$ if and only if $\gamma>\gamma_{0}$. Hence the solution $x_{\varepsilon}$ for $\varepsilon=1 / 3$ exists if $\gamma_{0}<\gamma<1 / 2$. Next, a straightforward but tedious calculation shows the right-hand side of (9.2) satisfies the integral equation (E). Finally, we note this function is in the cone $C^{a}$, so it must be the solution $x_{\varepsilon}$.

It is not too surprising that the explicit solution (9.2) can be given, for $x_{\varepsilon}$ can be obtained by solving an "integrable" ordinary differential equation when $\varepsilon=1 / 3$. Indeed, letting $y_{ \pm}(t)=x_{\varepsilon}(t \pm 2 / 3)$ we see from the differentiated form (1.5) of $(\mathrm{E})$ and from the periodicity that

$$
\left\{\begin{array}{l}
x_{\varepsilon}^{\prime}=\frac{3}{2}\left[f\left(y_{-}\right)-f\left(y_{+}\right)\right], \\
y_{-}^{\prime}=\frac{3}{2}\left[f\left(y_{+}\right)-f\left(x_{\varepsilon}\right)\right], \\
y_{+}^{\prime}=\frac{3}{2}\left[f\left(x_{\varepsilon}\right)-f\left(y_{-}\right)\right] .
\end{array}\right.
$$

The third order system (9.3) possesses the two integrals $I_{1}=x_{\varepsilon}+y_{-}+y_{+}$and $I_{2}=$ $F\left(x_{\varepsilon}\right)+F\left(y_{-}\right)+F\left(y_{+}\right)$, where the function $F$ is the primitive of $f$. Thus, at least in principle, (9.3) can be integrated by quadratures. Obtaining periodic solutions of a 
differential-delay equation by means of a related ordinary differential equation, in this fashion, was done previously by Kaplan and Yorke [13].

For the parameter values $\varepsilon=1 / 2$ and $\varepsilon=2 / 3$ completely integrable ordinary differential equations can be obtained in a similar fashion. These, perhaps, might prove useful in a stability analysis of $x_{\varepsilon}$ for these two values of $\varepsilon$ as our theory in Section 8 does not apply when $\varepsilon>1 / 3$.

PROPOSITION 9.5. For $\varepsilon=1 / 3, f=f^{0}$ and $\gamma-\gamma_{0}>0$ sufficiently small, the solution $x_{\varepsilon}$, given explicitly by (9.2), is asymptotically stable.

Proof. We fix $\varepsilon$ at $1 / 3$ and consider $\gamma$ as a Hopf bifurcation parameter. Indeed, a simple calculation shows that the roots $\mu(\gamma)$ and $\overline{\mu(\gamma)}$ of the characteristic equation (2.1), with $f^{\prime}(0)=-(1+\gamma), \gamma$ near $\gamma_{0}$, and $\mu\left(\gamma_{0}\right)=i \pi$, satisfy $\operatorname{Re} \mu^{\prime}\left(\gamma_{0}\right)>0$. Therefore the results of Theorem 11.2 of [4] apply and give the solution $x_{\varepsilon}(t)$ in (9.2) as the solution of this Hopf bifurcation problem. At the bifurcation point $\gamma=\gamma_{0}$ the multiplier $\alpha=1$ has multiplicity two, corresponding to the slowly oscillating solutions $\cos \pi t$ and $\sin \pi t$ of (LE). For $\gamma>\gamma_{0}$ the trivial multiplier $\alpha=1$ is simple; the other principal multiplier $\beta=\beta(\gamma)$ satisfies $\beta \neq 1$, and $\beta \rightarrow 1$ as $\gamma \downarrow \gamma_{0}$. The question is whether one has $\beta(\gamma)>1$ or $\beta(\gamma)<1$.

In Theorem 11.2 of [4] an explicit relation between the lowest order terms of the parameter $\gamma$ and multiplier $\beta$, expanded as functions of a perturbation parameter $\Theta$ near the bifurcation point, is given. Taking $\Theta=\left[(4 / 3)\left(1-\gamma_{0} / \gamma\right)\right]^{1 / 2}$ to be the amplitude we find that $\gamma(\Theta)=\gamma_{0}+(3 / 4) \gamma_{0} \Theta^{2}+o\left(\Theta^{2}\right)$. The results in [4] then give

$$
\begin{aligned}
\beta(\gamma(\Theta)) & =\exp \left[-3 \gamma_{0} \operatorname{Re} \mu^{\prime}\left(\gamma_{0}\right) \Theta^{2}\right]+o\left(\Theta^{2}\right) \\
& =1-3 \gamma_{0} \operatorname{Re} \mu^{\prime}\left(\gamma_{0}\right) \Theta^{2}+o\left(\Theta^{2}\right) .
\end{aligned}
$$

We conclude that $\beta(\gamma(\Theta))<1$ for $\Theta$ positive and small, hence $x_{\varepsilon}(t)$ is asymptotically stable.

Theorem 9.1 is now proved. Theorem 9.2 concerns the $2 / k$-periodic solution $x_{k \varepsilon}(k t)$ where either $\varepsilon<\varepsilon^{*} / k<1 / 3$ or $\varepsilon \in\left(\varepsilon_{n}^{-} / k, \varepsilon_{n}^{+} / k\right) \cap(0,1 / 3]$. In either case $k \geqslant 2$ and so the solution $x_{k \varepsilon}(k t)$ has consecutive zeros spaced a distance $1 / k \leqslant 1 / 2$ apart. As $1 / 2<1-\varepsilon$, it follows that the solution $z(t)=x_{k \varepsilon}^{\prime}(k t)$ of the linearized equation

$$
z(t)=\frac{1}{2 \varepsilon} \int_{1-\varepsilon}^{1+\varepsilon} f^{\prime}\left(x_{k \varepsilon}(k t-k \tau)\right) z(t-\tau) d \tau
$$

is never slowly oscillating; thus the trivial multiplier $\alpha=1$ of $(9.4)$ is not a principal multiplier.

Consider now equation (9.4) when $\varepsilon$ assumes the value $\varepsilon^{*} / k$ or $\varepsilon_{n}^{-} / k$ corresponding to the Hopf bifurcation. In case $\varepsilon=\varepsilon_{n}^{-} / k$, let us assume this value satisfies $\varepsilon_{n}^{-} / k<1 / 3$. In either case, the kernel of (9.4) is simply the constant $f^{\prime}(0)$ so that equation (9.4) is autonomous. In addition, $\alpha=1$ is a multiplier but not a principal multiplier. Lemma 2.3 implies that $\operatorname{dim} \mathscr{H}_{*}=2$ for this equation, as the two roots in the strip (2.3) give rise to principal multipliers. Moreover, these principal multipliers 
satisfy $\left|\alpha_{1}\right| \geqslant\left|\alpha_{2}\right|>1$ because the multiplier $\alpha=1$ is not principal. Finally, we see from Propositions 8.7 and 8.8 that this situation is maintained as $\varepsilon$ varies throughout the interval $\left(0, \varepsilon^{*} / k\right) \subseteq(0,1 / 3)$ or $\left(\varepsilon_{n}^{-} / k, \varepsilon_{n}^{+} / k\right) \cap(0,1 / 3]$. This completes the proof of Theorem 9.2.

\section{Concluding Remarks and Open Problems}

Observe that if $x: R \rightarrow R$ is any (discontinuous) function satisfying

$$
\begin{array}{cc}
x(t+1)=-x(t) & \text { for all } t, \\
x(t) \in\{-1,1\} & \text { for all } t
\end{array}
$$

then $x$ is a solution of the difference equation (D). Obviously there are uncountably many such functions even after we identify functions $x(t)$ and $x\left(t+t_{0}\right)$ differing merely by a time translation. Our results indicate that the integral equation (E), considered as a perturbation of (D), selects a countable subfamily of these solutions as limits of periodic solutions arising from Hopf bifurcations. That is, only the functions

$$
x(t)=\operatorname{sqw}(k t), \quad k=1,3,5, \cdots,
$$

with discontinuities spaced equally a distance $1 / k$ apart occur as limits of the periodic solutions of (D) considered. This raises the question of whether there exist other branches of periodic solutions of (E), not arising from Hopf bifurcations, with limiting behaviour as $\varepsilon \downarrow 0$ described by other members of the family (10.1). More precisely, given numbers

$$
0=\alpha_{0}<\alpha_{1}<\alpha_{2}<\cdots<\alpha_{k}=1,
$$

where $k$ is odd, consider the function $v$ defined by

$$
\begin{array}{ll}
v(t)=(-1)^{j}, & \text { if } \alpha_{j} \leqslant t<\alpha_{j+1}, \\
v(t+1)=-v(t), & \text { for all } t .
\end{array}
$$

Does there exist, for small $\varepsilon$, a solution $x(t)$ of (E) which approaches $v(t)$ as $\varepsilon \downarrow 0$, and which has appropriate transition layers near the discontinuities $\alpha_{j}$ of $v$ ? Must such a solution (if it exists) have period exactly two or does its period merely approach two at some rate as $\varepsilon \downarrow 0$ ?

Apart from the local results on stability obtained here, virtually nothing is known about the global dynamical behaviour of solutions of initial value problems of (E). Is it true that any solution $x(t)$ of an initial value problem must approach either a periodic solution or else the qrigin as $t \rightarrow \infty$ ? Is this at least true for slowly oscillating solutions? Does the phase space of $(\mathrm{E})$ admit a Morse Decomposition based on rates of oscillation of solutions as is the case (see $[17,18]$ ) for certain differential-delay equations?

Finally, we ask to what extent our results depend on the monotonicity, convexity and symmetry hypotheses on the nonlinearity $f$. Certainly these are essential in 
proving the very rich structure of the solution branches obtained here. If some of the hypotheses are relaxed, does there still exist a continuum of periodic solutions (but not necessarily a smooth curve of solutions) extending from the Hopf bifurcation at $\varepsilon^{*}$ to $\varepsilon=0$ ? This is true for a class of singularly perturbed differential-delay equations $[19,20]$

$$
\varepsilon x^{\prime}(t)=-x(t)+f(x(t-1))
$$

under certain conditions of $f$, notably that the period two orbit $\{-1,1\}$ of the difference equation (D) attracts all orbits starting from a nonzero initial condition. Walther [30] has shown that secondary bifurcations can occur. If $f$ is not monotone do chaotic solutions exist? Again this seems to be true from numerical evidence $[5,6]$ for equation (10.2); rigorous results for special cases of this and other equations are also available $[11,27,29]$.

\section{References}

[1] S.-N. Chow and J. Mallet-Paret, The Fuller index and global Hopf bifurcation. J. Differential Equations, 29 (1978), 66-85.

[2] S.-N. Chow and J. Mallet-Paret, Singularly perturbed delay-differential equations. Coupled Nonlinear Oscillators (eds. J. Chandra and A. C. Scott), North-Holland Math. Studies, 80, 1983, $7-12$.

[3] J. M. Cushing, Nontrivial periodic solutions of some Volterra integral equations. Volterra Equations (eds. S. O. Londen and O. Staffans), Lecture Notes in Math. 737, Springer, 1979, 50-66.

[4] O. Diekmann and S. A. van Gils, Invariant manifolds for Volterra integral equations of convolution type. J. Differential Equations, 54 (1984), 139-180.

[5] J. D. Farmer, Chaotic attractors of an infinite-dimensional dynamical system. Physica, 4D (1982), 366-393.

[6] L. Glass and M. Mackey, Oscillation and chaos in physiological control systems. Science, 197 (1977), 287-289.

[ 7] J. M. Greenberg, Periodic solutions to a population equation. Dynamical Systems (eds. L. Cesari, J. K. Hale and J. P. La Salle), Vol. II, Academic Press, New York, 1976, 153-157.

[8] K. P. Hadeler and J. Tomiuk, Periodic solutions of difference-differential equations. Arch. Rational Mech. Anal., 65 (1977), 87-95.

[9] J. K. Hale, Theory of Functional Differential Equations. Springer, 1977.

[10] J. K. Hale, Nonlinear oscillations in equations with delays. Nonlinear Oscillations in Biology (ed. F. C. Hoppensteadt) AMS Lectures in Applied Math., 17, 1978, 157-186.

[11] U. an der Heiden and H.-O. Walther, Existence of chaos in control systems with delayed feedback. J. Differential Equations, 47 (1983), 273-295.

[12] F. C. Hoppensteadt, Perturbation methods in biology. Mathematics of Biology (ed. M. Iannelli), C.I.M.E., 1979, 265-322.

[13] J. L. Kaplan and J. A. Yorke, Ordinary differential equations which yield periodic solutions of differential delay equations. J. Math. Anal. Appl., 48 (1974), 317-324.

[14] J. L. Kaplan and J. A. Yorke, On the stability of a periodic solution of a differential delay equation. SIAM J. Math. Anal., 6 (1975), 268-282.

[15] J. L. Kaplan and J. A. Yorke, On the nonlinear differential delay equation $x^{\prime}(t)=f(x(t), x(t-1))$. J. Differential Equations, 23 (1977), 293-314.

[16] M. A. Krasnosel'skii, Positive Solutions of Operator Equations. Noordhoff, Groningen, 1964.

[17] J. Mallet-Paret, Morse decompositions and global continuation of periodic solutions for singularly perturbed delay equations. Systems of Nonlinear Partial Differential Equations (ed. J. M. Ball), 
Reidel, Dordrecht, 1983, 351-365.

[18] J. Mallet-Paret, Morse decompositions for delay differential equations. In preparation.

[19] J. Mallet-Paret and R. D. Nussbaum, Global continuation and complicated trajectories for periodic solutions of a differentiat-delay equation. To appear in: Proc. Symposia Pure Math., Amer. Math. Soc.

[20] J. Mallet-Paret and R. D. Nussbaum, Global continuation and asymptotic behaviour for periodic solutions of a differential-delay equation. In preparation.

[21] J. Mallet-Paret and J. A. Yorke, Two types of Hopf bifurcation points: sources and sinks of families of periodic orbits. Ann. N. Y. Acad. Sci., 357 (1980), 300-304.

[22] J. Mallet-Paret and J. A. Yorke, Snakes: oriented families of periodic orbits, their sources, sinks and continuation. J. Differential Equations, 43 (1982), 419-450.

[23] M. Martelli, K. Schmitt and H. Smith, Periodic solutions of some nonlinear delay-differential equations. J. Math. Anal. Appl., 74 (1980), 494-503.

[24] A. D. Myshkis, Lineare Differentialgleichungen mit nacheilendem Argument. Deutscher Verlag Wiss., Berlin, 1955.

[25] R. D. Nussbaum, A global bifurcation theorem with applications to functional differential equations, J. Funct. Anal., 19 (1975), 319-338.

[26] R. D. Nussbaum, Periodic solutions of nonlinear autonomous functional differential equations. Functional Differential Equations and Approximation of Fixed Points (eds. H.-O. Peitgen and H.-O. Walther) Lecture Notes in Math. 730, Springer, 1979, 283-325.

[27] H. Peters, Comportement chaotique d'une équation différentielle retardée. C. R. Acad. Sci. Paris, Ser. A, 290 (1980), 1119-1122.

[28] H.-O. Walther, On instability, $\omega$-limit sets and periodic solutions to nonlinear autonomous differential delay equations. Functional Differential Equations and Approximation of Fixed Points (eds. H.-O. Peitgen and H.-O. Walther) Lecture Notes in Math. 730, Springer, 1979, 489-503.

[29] H.-O. Walther, Homoclinic solution and chaos in $\dot{x}(t)=f(x(t-1))$. Nonlinear Anal. Theory Methods Appl., 5 (1981), 775-788.

[30] H.-O. Walther, Bifurcation from periodic solutions in functional differential equations. Math. Z., 182 (1983), 269-289.

[31] R. D. Nussbaum, Periodic solutions of special differential equations: an example in non-linear functional analysis. Proc. Roy. Soc. Edinburgh, 81A (1978), 131-151. 